Pacific

Journal of

Mathematics

ASYMPTOTIC BEHAVIOR OF PALAIS-SMALE SEQUENCES ASSOCIATED WITH

FRACTIONAL YAMABE-TYPE EQUATIONS

Yi FANG AND MARÍA DEL MAR GONZÁLEZ 


\title{
ASYMPTOTIC BEHAVIOR OF PALAIS-SMALE SEQUENCES ASSOCIATED WITH FRACTIONAL YAMABE-TYPE EQUATIONS
}

\author{
Yi FANG AND MARÍA DEL MAR GONZÁLEZ
}

\begin{abstract}
In this paper, we analyze the asymptotic behavior of Palais-Smale sequences associated to fractional Yamabe-type equations on an asymptotically hyperbolic Riemannian manifold. We prove that Palais-Smale sequences can be decomposed into the solution of the limit equation plus a finite number of bubbles, which are the rescaling of the fundamental solution for the fractional Yamabe equation on Euclidean space. We also verify the noninterfering fact for multibubbles.
\end{abstract}

\section{Introduction and statement of results}

Let $\Omega$ be a smooth bounded domain in $\mathbb{R}^{n}, n \geq 3$. Fix a constant $\lambda$, and consider the Dirichlet boundary value problem of the elliptic PDE

$$
\begin{cases}-\Delta u-\lambda u=u|u|^{\frac{4}{n-2}} & \text { in } \Omega, \\ u=0 & \text { on } \partial \Omega .\end{cases}
$$

The associated variational functional of (1-1) in the Sobolev space $W_{0}^{1,2}(\Omega)$ is

$$
E(u)=\frac{1}{2} \int_{\Omega}\left(|\nabla u|^{2}-\lambda u^{2}\right) d x-\frac{n-2}{2 n} \int_{\Omega}|u|^{\frac{2 n}{n-2}} d x .
$$

Suppose that the sequence $\left\{u_{\alpha}\right\}_{\alpha \in \mathbb{N}} \subset W_{0}^{1,2}(\Omega)$ satisfies the Palais-Smale condition, $\left\{E\left(u_{\alpha}\right)\right\}_{\alpha \in \mathbb{N}}$ is uniformly bounded and $D E\left(u_{\alpha}\right) \rightarrow 0$, strongly in $\left(W_{0}^{1,2}(\Omega)\right)^{\prime}$, as $\alpha \rightarrow+\infty$, where $\left(W_{0}^{1,2}(\Omega)\right)^{\prime}$ is the dual space of $W_{0}^{1,2}(\Omega)$. In an elegant paper, M. Struwe [1984] considered the asymptotic behavior of $\left\{u_{\alpha}\right\}_{\alpha \in \mathbb{N}}$. In fact, in

Fang is fully supported by China Scholarship Council (CSC) for visiting the University of California, Santa Cruz. González is supported by Spain Government projects MTM2011-27739-C04-01 and MTM2014-52402-C3-1-P, and GenCat 2009SGR345.

MSC2010: primary 35J70; secondary 58J32.

Keywords: fractional Yamabe problem, Palais-Smale sequences. 
the $W_{0}^{1,2}(\Omega)$ norm, $u_{\alpha}$ can be approximated by the solution to (1-1) plus a finite number of bubbles, which are the rescaling of the nontrivial entire solution of

$$
-\Delta u=u|u|^{\frac{4}{n-2}} \text { in } \mathbb{R}^{n} \text { and } \quad u(x) \rightarrow 0 \text { as }|x| \rightarrow+\infty .
$$

One may pose the analogous problem on a manifold. Let $\left(M^{n}, g\right)$ be a smooth compact Riemannian manifold without boundary. Consider a sequence of elliptic PDEs like

$$
-\Delta_{g} u+h_{\alpha} u=u^{\frac{n+2}{n-2}},
$$

where $\alpha \in \mathbb{N}$ and $\Delta_{g}$ denotes the Laplace-Beltrami operator of the metric $g$. Assume that $h_{\alpha}$ satisfies the condition that there exists $C>0$ with $\left|h_{\alpha}(x)\right| \leq C$ for any $\alpha$ and any $x \in M$; also $h_{\alpha} \rightarrow h_{\infty}$ in $L^{2}(M)$ as $\alpha \rightarrow+\infty$. The limit equation is denoted by

$$
-\Delta_{g} u+h_{\infty} u=u^{\frac{n+2}{n-2}} .
$$

The related variational functional for $\left(E_{\alpha}\right)$ is

$$
E_{g}^{\alpha}(u)=\frac{1}{2} \int_{M}|\nabla u|_{g}^{2} d v_{g}+\frac{1}{2} \int_{M} h_{\alpha} u^{2} d v_{g}-\frac{n-2}{2 n} \int_{M}|u|^{\frac{2 n}{n-2}} d v_{g} .
$$

Suppose that $\left\{u_{\alpha} \geq 0\right\}_{\alpha \in \mathbb{N}} \subset W^{1,2}(M)$ also satisfies the Palais-Smale condition. O. Druet, E. Hebey, and F. Robert [Druet et al. 2004] proved that, in the $W^{1,2}(M)$ sense, $u_{\alpha}$ can be decomposed into the solution of $\left(E_{\infty}\right)$ plus a finite number of bubbles, which are the rescaling of the nontrivial solution of

$$
-\Delta u=u^{\frac{n+2}{n-2}} \text { in } \mathbb{R}^{n} \text {. }
$$

Let $\left(M^{n}, g\right)$ be a compact Riemannian manifold with boundary $\partial M$. Recently, S. Almaraz [2014] considered the following sequence of equations with nonlinear boundary value condition:

$$
\begin{cases}-\Delta_{g} u=0 & \text { in } M, \\ -\frac{\partial}{\partial \eta_{g}} u+h_{\alpha} u=u^{\frac{n}{n-2}} & \text { on } \partial M,\end{cases}
$$

where $\alpha \in \mathbb{N}$ and $\eta_{g}$ is the inward unit normal vector to $\partial M$. The associated energy functional for (1-2) is

$$
\bar{E}_{g}^{\alpha}(u)=\frac{1}{2} \int_{M}|\nabla u|_{g}^{2} d v_{g}+\frac{1}{2} \int_{\partial M} h_{\alpha} u^{2} d \sigma_{g}-\frac{n-2}{2(n-1)} \int_{\partial M}|u|^{\frac{2(n-1)}{n-2}} d \sigma_{g},
$$

for $u \in H^{1}(M):=\left\{u \mid \nabla u \in L^{2}(M), u \in L^{2}(\partial M)\right\}$. Here $d v_{g}$ and $d \sigma_{g}$ are the volume forms of $M$ and $\partial M$, respectively. He also showed that a nonnegative Palais-Smale sequence $\left\{u_{\alpha}\right\}_{\alpha \in \mathbb{N}}$ of $\left\{\bar{E}_{g}^{\alpha}\right\}_{\alpha \in \mathbb{N}}$ converges, in the $H^{1}(M)$ sense, to 
a solution of the limit equation (the equation replacing $h_{\alpha}$ by $h_{\infty}$ in (1-2)) plus a finite number of bubbles.

Motivated by these facts and the original study of the fractional Yamabe problem by M.d.M. González and J. Qing [2013] (see also [González 2015]), in this paper we shall be interested in the asymptotic behavior of nonnegative Palais-Smale sequences associated with the fractional Yamabe equation on an asymptotically hyperbolic Riemannian manifold.

Let $\left(X^{n+1}, g^{+}\right), n \geq 3$, be a smooth Riemannian manifold with smooth boundary $\partial X^{n+1}=M^{n}$. A function $\rho_{*}$ is called a defining function of the boundary $M^{n}$ in $X^{n+1}$ if it satisfies

$$
\rho_{*}>0 \text { in } X^{n+1}, \quad \rho_{*}=0 \text { on } M^{n}, \quad d \rho_{*} \neq 0 \text { on } M^{n} .
$$

We say that a metric $g^{+}$is conformally compact if there exists a defining function $\rho_{*}$ such that $\left(\overline{X^{n+1}}, \bar{g}_{*}\right)$ is compact for $\bar{g}_{*}=\rho_{*}^{2} g^{+}$. This induces a conformal class of metrics $\hat{h}=\left.\bar{g}_{*}\right|_{M^{n}}$ when defining functions vary. The conformal manifold $\left(M^{n},[\hat{h}]\right)$ is called the conformal infinity of $\left(X^{n+1}, g^{+}\right)$. A metric $g^{+}$is said to be asymptotically hyperbolic if it is conformally compact and the sectional curvature approaches -1 at infinity. It is easy to check then that $\left|d \rho_{*}\right|_{\bar{g}_{*}}^{2}=1$ on $M^{n}$.

Using the meromorphic family of scattering operators $S(s)$ introduced by C.R. Graham and M. Zworski [2003], we will define the so-called fractional order scalar curvature. Given an asymptotically hyperbolic Riemannian manifold $\left(X^{n+1}, g^{+}\right)$ and a representative $\hat{h}$ of the conformal infinity $\left(M^{n},[\hat{h}]\right)$, there is a unique geodesic defining function $\rho_{*}$ such that, in $M^{n} \times(0, \delta)$ in $X^{n+1}$ for small $\delta, g^{+}$has the normal form

$$
g^{+}=\rho_{*}^{-2}\left(d \rho_{*}^{2}+h_{\rho_{*}}\right),
$$

where $h_{\rho_{*}}$ is a one parameter family of metric on $M^{n}$ such that

$$
h_{\rho_{*}}=\hat{h}+h^{(1)} \rho_{*}+O\left(\rho_{*}^{2}\right) .
$$

It is well-known [Graham and Zworski 2003] that, given $f \in \mathbb{C}^{\infty}\left(M^{n}\right)$ and $s \in \mathbb{C}$, $\operatorname{Re}(s)>n / 2$ and $s(n-s)$ is not an $L^{2}$ eigenvalue for $-\Delta_{g}+$, then the generalized eigenvalue problem

$$
-\Delta_{g}+\tilde{u}-s(n-s) \tilde{u}=0 \quad \text { in } X^{n+1}
$$

has a solution of the form

$$
\tilde{u}=F\left(\rho_{*}\right)^{n-s}+G\left(\rho_{*}\right)^{s}, \quad F, G \in \mathscr{C}^{\infty}\left(\overline{X^{n+1}}\right),\left.\quad F\right|_{\rho_{*}=0}=f .
$$

The scattering operator on $M^{n}$ is then defined as

$$
S(s) f=\left.G\right|_{M^{n}} .
$$


Now we consider the normalized scattering operators

$$
P_{\gamma}\left[g^{+}, \hat{h}\right]=d_{\gamma} S\left(\frac{n}{2}+\gamma\right), \quad d_{\gamma}=2^{2 \gamma} \frac{\Gamma(\gamma)}{\Gamma(-\gamma)} .
$$

Note that $P_{\gamma}\left[g^{+}, \hat{h}\right]$ is a pseudodifferential operator whose principal symbol is equal to the one of $(-\Delta \hat{h})^{\gamma}$. Moreover, $P_{\gamma}\left[g^{+}, \hat{h}\right]$ is conformally covariant, i.e., for any $\varphi, w \in \mathscr{C}^{\infty}\left(\overline{X^{n+1}}\right)$ and $w>0$,

$$
P_{\gamma}\left[g^{+}, w^{\frac{4}{n-2 \gamma}} \hat{h}\right](\varphi)=w^{-\frac{n+2 \gamma}{n-2 \gamma}} P_{\gamma}\left[g^{+}, \hat{h}\right](w \varphi) .
$$

Thus we shall call $P_{\gamma}\left[g^{+}, \hat{h}\right]$ the conformal fractional Laplacian for any $\gamma \in(0, n / 2)$ such that $n^{2} / 4-\gamma^{2}$ is not an $L^{2}$ eigenvalue for $-\Delta_{g+}$.

The fractional scalar curvature associated to the operator $P_{\gamma}\left[g^{+}, \hat{h}\right]$ is defined as

$$
Q_{\gamma}^{\hat{h}}=P_{\gamma}\left[g^{+}, \hat{h}\right](1) \text {. }
$$

The scattering operator has a pole at the integer values $\gamma$. However, in such cases the residue may be calculated and, in particular, when $g^{+}$is Poincaré-Einstein metric, for $\gamma=1$,

$$
P_{1}\left[g^{+}, \hat{h}\right]=-\Delta \hat{h}+\frac{n-2}{4(n-1)} R \hat{h},
$$

which is exactly the so-called conformal Laplacian, and

$$
Q_{1}^{\hat{h}}=\frac{n-2}{4(n-1)} R \hat{h} .
$$

Here, $R_{\hat{h}}$ is the scalar curvature of the metric $\hat{h}$.

For $\gamma=2, P_{2}\left[g^{+}, \hat{h}\right]$ is precisely the Paneitz operator and its associated curvature is known as $Q$-curvature [2008]. In general, $P_{k}\left[g^{+}, \hat{h}\right]$ for $k \in \mathbb{N}$ are precisely the conformal powers of the Laplacian studied in [Graham et al. 1992].

We consider the conformal change $\hat{h}_{w}=w^{4 /(n-2 \gamma)} \hat{h}$ for some $w>0$; then by (1-4),

$$
P_{\gamma}\left[g^{+}, \hat{h}\right](w)=Q_{\gamma}^{\hat{h}_{w}} w^{\frac{n+2 \gamma}{n-2 \gamma}} \quad \text { in }\left(M^{n}, \hat{h}\right) .
$$

If for this conformal change $Q_{\gamma}^{\hat{h}_{w}}$ is a constant $C_{\gamma}$ on $M^{n}$, this problem reduces to

$$
P_{\gamma}\left[g^{+}, \hat{h}\right](w)=C_{\gamma} w^{\frac{n+2 \gamma}{n-2 \gamma}} \quad \text { in }\left(M^{n}, \hat{h}\right),
$$

which is the so-called fractional Yamabe equation or the $\gamma$-Yamabe equation, studied in [González and Qing 2013].

Throughout the paper, we always suppose that $\gamma \in(0,1)$, and such that the first eigenvalue for $-\Delta_{g}+$ satisfies $\lambda_{1}>n^{2} / 4-\gamma^{2}$, as was pointed out in [Case and Chang 2015; Case 2015]. 
It is well known that the above fractional Yamabe equation may be rewritten as a degenerate elliptic Dirichlet-to-Neumann boundary problem. For that, we first recall some results obtained by Chang and González in [2011] (see also the paper by J. Case and S.A. Chang [2015]). Suppose that $u^{*}$ solves

$$
\begin{cases}-\Delta_{g}+u^{*}-s(n-s) u^{*}=0 & \text { in } X^{n+1}, \\ \lim _{\rho_{*} \rightarrow 0} \rho_{*}^{s-n} u^{*}=1 & \text { on } M^{n} .\end{cases}
$$

Proposition 1.1 [Chang and González 2011; González and Qing 2013]. Suppose that $f \in \mathscr{C}^{\infty}(M)$. Assume that $\tilde{u}, u^{*}$ are solutions to (1-3) and (1-6), respectively. Then $\rho=\left(u^{*}\right)^{1 /(n-s)}$ is a geodesic defining function. Moreover, $u=\tilde{u} / u^{*}=\rho^{s-n} \tilde{u}$ solves

$$
\begin{cases}-\operatorname{div}\left(\rho^{1-2 \gamma} \nabla u\right)=0 & \text { in } X^{n+1}, \\ u=f & \text { on } M^{n}\end{cases}
$$

with respect to the metric $g=\rho^{2} g^{+}$, and $u$ is the unique minimizer of the energy functional

$$
I(v)=\int_{X^{n+1}} \rho^{1-2 \gamma}|\nabla v|_{g}^{2} d v_{g}
$$

among all the extensions $v \in W^{1,2}\left(X^{n+1}, \rho^{1-2 \gamma}\right)$ (see Definition 2.1) satisfying $\left.v\right|_{M^{n}}=f$. Moreover,

$$
\rho=\rho_{*}\left(1+\frac{Q_{\gamma}^{\hat{h}}}{(n-s) d_{\gamma}} \rho_{*}^{2 \gamma}+O\left(\rho_{*}^{2}\right)\right)
$$

near the conformal infinity and

$$
P_{\gamma}\left[g^{+}, \hat{h}\right](f)=-d_{\gamma}^{*} \lim _{\rho \rightarrow 0} \rho^{1-2 \gamma} \partial_{\rho} u+Q_{\gamma}^{\hat{h}} f, \quad d_{\gamma}^{*}=-\frac{d_{\gamma}}{2 \gamma}>0,
$$

provided that $\operatorname{Tr} \hat{h} h^{(1)}=0$ when $\gamma \in\left(\frac{1}{2}, 1\right)$. Here $\left.g\right|_{M^{n}}=\hat{h}$, and has asymptotic expansion

$$
g=d \rho^{2}\left[1+O\left(\rho^{2 \gamma}\right)\right]+\hat{h}\left[1+O\left(\rho^{2 \gamma}\right)\right] .
$$

We fix $\gamma \in(0,1)$. By Proposition 1.1, one can rewrite the fractional Yamabe equation (1-5) into the following problem:

$$
\begin{cases}-\operatorname{div}\left(\rho^{1-2 \gamma} \nabla u\right)=0 & \text { in }\left(X^{n+1}, g\right), \\ u=w & \text { on }\left(M^{n}, \hat{h}\right), \\ -d_{\gamma}^{*} \lim _{\rho \rightarrow 0} \rho^{1-2 \gamma} \partial_{\rho} u+Q_{\gamma}^{\hat{h}} w=C_{\gamma} w^{\frac{n+2 \gamma}{n-2 \gamma}} & \text { on }\left(M^{n}, \hat{h}\right) .\end{cases}
$$

In this paper we consider the positive curvature case $C_{\gamma}>0$. Without loss of generality, we assume that $C_{\gamma}=d_{\gamma}^{*}$. 
In the particular case $\gamma=\frac{1}{2}$, one may check that (1-8) reduces to (1-2), which was considered in [Almaraz 2014]. The main difficulty we encounter here is the presence of the weight that makes the extension equation only degenerate elliptic.

Next, we introduce the so-called $\gamma$-Yamabe constant [González and Qing 2013]. For the defining function $\rho$ mentioned above, we set

$$
I_{\gamma}[u, g]=\frac{d_{\gamma}^{*} \int_{X} \rho^{1-2 \gamma}|\nabla u|_{g}^{2} d v_{g}+\int_{M} Q_{\gamma}^{\hat{h}} u^{2} d \sigma_{\hat{h}}}{\left(\int_{M}|u|^{2^{*}} d \sigma_{\hat{h}}\right)^{\frac{2}{2^{*}}}},
$$

then the $\gamma$-Yamabe constant is defined as

$$
\Lambda_{\gamma}(M,[\hat{h}])=\inf \left\{I_{\gamma}[u, g] \mid u \in W^{1,2}\left(X, \rho^{1-2 \gamma}\right)\right\} .
$$

It was shown in [loc. cit.] that in the positive curvature case $C_{\gamma}>0$ we must have $\Lambda_{\gamma}(M,[\hat{h}])>0$.

Now we take a perturbation of the linear term $Q_{\gamma}^{\hat{h}} w$ to a general $-d_{\gamma}^{*} Q_{\alpha}^{\gamma} w$, where $Q_{\alpha}^{\gamma} \in \mathscr{C}^{\infty}\left(M^{n}\right), \alpha \in \mathbb{N}$. Suppose that for any $\alpha \in \mathbb{N}$ and any $x \in M^{n}$, there exists a constant $C>0$ such that $\left|Q_{\alpha}^{\gamma}(x)\right| \leq C$. Also assume that $Q_{\alpha}^{\gamma} \rightarrow Q_{\infty}^{\gamma}$ in $L^{2}\left(M^{n}, \hat{h}\right)$ as $\alpha \rightarrow+\infty$. We will consider a family of equations

$$
\begin{cases}-\operatorname{div}\left(\rho^{1-2 \gamma} \nabla u\right)=0 & \text { in }\left(X^{n+1}, g\right), \\ u=w & \text { on }\left(M^{n}, \hat{h}\right), \\ -\lim _{\rho \rightarrow 0} \rho^{1-2 \gamma} \partial_{\rho} u+Q_{\alpha}^{\gamma} w=w^{\frac{n+2 \gamma}{n-2 \gamma}} & \text { on }\left(M^{n}, \hat{h}\right) .\end{cases}
$$

The associated variational functional to (1-10) is

$$
\begin{aligned}
I_{g}^{\gamma, \alpha}(u)=\frac{1}{2} \int_{X^{n+1}} \rho^{1-2 \gamma} & |\nabla u|_{g}^{2} d v_{g} \\
& +\frac{1}{2} \int_{M^{n}} Q_{\alpha}^{\gamma} u^{2} d \sigma_{\hat{h}}-\frac{n-2 \gamma}{2 n} \int_{M^{n}}|u|^{\frac{2 n}{n-2 \gamma}} d \sigma_{\hat{h}} .
\end{aligned}
$$

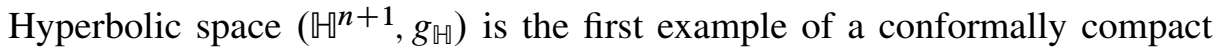
Einstein manifold. As $\left(\mathbb{T}^{n+1}, g_{\mathbb{H}}\right)$ can be characterized as the upper half-space $\mathbb{R}_{+}^{n+1}$ endowed with metric $g^{+}=y^{-2}\left(|d x|^{2}+d y^{2}\right)$, where $x \in \mathbb{R}^{n}, y \in \mathbb{R}_{+}$, then the Dirichlet-to-Neumann problem (1-8) reduces to

$$
\begin{cases}-\operatorname{div}\left(y^{1-2 \gamma} \nabla u\right)=0 & \text { in }\left(\mathbb{R}_{+}^{n+1},|d x|^{2}+d y^{2}\right), \\ u=w & \text { on }\left(\mathbb{R}^{n},|d x|^{2}\right), \\ -\lim _{y \rightarrow 0} y^{1-2 \gamma} \partial_{y} u=w^{\frac{n+2 \gamma}{n-2 \gamma}} & \text { on }\left(\mathbb{R}^{n},|d x|^{2}\right) .\end{cases}
$$

And the variational functional to (1-12) is defined as

$$
\widetilde{E}(u)=\frac{1}{2} \int_{\mathbb{R}_{+}^{n+1}} y^{1-2 \gamma}|\nabla u(x, y)|^{2} d x d y-\frac{n-2 \gamma}{2 n} \int_{\mathbb{R}^{n}}|u(x, 0)|^{\frac{2 n}{n-2 \gamma}} d x .
$$


Up to multiplicative constants, the only solution to problem (1-12) is given by the standard

$$
w(x)=w_{a}^{\lambda}(x)=\left(\frac{\lambda}{|x-a|^{2}+\lambda^{2}}\right)^{\frac{n-2 \gamma}{2}}
$$

for some $a \in \mathbb{R}^{n}$ and $\lambda>0$ [González and Qing 2013; Jin et al. 2014]. By the Poisson formula of L. Caffarelli and L. Silvestre [2007], the corresponding extension can be expressed as

$$
U_{a}^{\lambda}(x, y)=\int_{\mathbb{R}^{n}} \frac{y^{2 \gamma}}{\left(|x-\xi|^{2}+y^{2}\right)^{(n+2 \gamma) / 2}} w_{a}^{\lambda}(\xi) d \xi .
$$

Here $U_{a}^{\lambda}$ is called a "bubble". Note that all of them have constant energy.

Remark 1.2. For any $a \in \mathbb{R}^{n}$ and $\lambda>0$, we have

$$
\widetilde{E}\left(U_{a}^{\lambda}\right)=\widetilde{E}\left(U_{0}^{1}\right)=\frac{\gamma}{n} \int_{\mathbb{R}^{n}}\left|U_{0}^{1}(x, 0)\right|^{\frac{2 n}{n-\gamma}} d x .
$$

Now we give some notations which will be used in the following. In the half space $\mathbb{R}_{+}^{n+1}=\left\{(x, y)=\left(x^{1}, \ldots, x^{n}, y\right) \in \mathbb{R}^{n+1} \mid y>0\right\}$ we define, for $r>0$,

$$
\begin{aligned}
B_{r}^{+}\left(z_{0}\right) & =\left\{z \in \mathbb{R}_{+}^{n+1}|| z-z_{0} \mid<r, z_{0} \in \mathbb{R}_{+}^{n+1}\right\}, \\
D_{r}\left(x_{0}\right) & =\left\{x \in \mathbb{R}^{n}|| x-x_{0} \mid<r, x_{0} \in \mathbb{R}^{n}\right\}, \\
\partial^{\prime} B_{r}^{+}\left(z_{0}\right) & =B_{r}^{+}\left(z_{0}\right) \cap \mathbb{R}^{n}, \\
\partial^{+} B_{r}^{+}\left(z_{0}\right) & =\partial B_{r}^{+}\left(z_{0}\right) \cap \mathbb{R}_{+}^{n+1} .
\end{aligned}
$$

Fix $\gamma \in(0,1)$. Suppose that $\left(X, g^{+}\right)$is an asymptotically hyperbolic manifold with boundary $M$ satisfying, in addition, $\operatorname{Tr} \hat{h} h^{(1)}=0$ when $\gamma \in(1 / 2,1)$. Let $\rho$ be the special defining function given in Proposition 1.1 and set $g=\rho^{2} g^{+}$and $\hat{h}=\left.g\right|_{M}$. Also, define

$$
\begin{aligned}
& \mathfrak{B}_{r}^{+}\left(z_{0}\right)=\left\{z \in X \mid d_{g}\left(z, z_{0}\right)<r, z_{0} \in \bar{X}\right\}, \\
& \mathfrak{D}_{r}\left(x_{0}\right)=\left\{x \in M \mid d \hat{h}\left(x, x_{0}\right)<r, x_{0} \in M\right\},
\end{aligned}
$$

Now, modulo the definitions of the weighted Sobolev space $W^{1,2}\left(X, \rho^{1-2 \gamma}\right)$ and of a Palais-Smale sequence (see Section 2), the main result of this paper is the following fractional type blow up analysis theorem:

Theorem 1.3. Let $\left\{u_{\alpha} \geq 0\right\}_{\alpha \in \mathbb{N}} \subset W^{1,2}\left(X, \rho^{1-2 \gamma}\right)$ be a Palais-Smale sequence for $\left\{I_{g}^{\gamma, \alpha}\right\}_{\alpha \in \mathbb{N}}$. Then there exists an integer $m \geq 1$, sequences $\left\{\mu_{\alpha}^{j}>0\right\}_{\alpha \in \mathbb{N}}$ and $\left\{x_{\alpha}^{j}\right\}_{\alpha \in \mathbb{N}} \subset M$ for $j=1, \ldots, m$, a nonnegative solution $u^{0} \in W^{1,2}\left(X, \rho^{1-2 \gamma}\right)$ to (2-4) and nontrivial nonnegative functions $U_{a_{j}}^{\lambda_{j}} \in W^{1,2}\left(\mathbb{R}_{+}^{n+1}, y^{1-2 \gamma}\right)$ for some $\lambda_{j}>0$ and $a_{j} \in \mathbb{R}^{n}$ as given in (1-13), satisfying, up to a subsequence, 
(1) $\mu_{\alpha}^{j} \rightarrow 0$ as $\alpha \rightarrow+\infty$, for $j=1, \ldots, m$;

(2) $\left\{x_{\alpha}^{j}\right\}_{\alpha \in \mathbb{N}}$ converges on $M$ as $\alpha \rightarrow+\infty$, for $j=1, \ldots, m$;

(3) As $\alpha \rightarrow+\infty$,

$$
\left\|u_{\alpha}-u^{0}-\sum_{j=1}^{m} \eta_{\alpha}^{j} u_{\alpha}^{j}\right\|_{W^{1,2}\left(X, \rho^{1-2 \gamma}\right)} \rightarrow 0,
$$

where

$$
u_{\alpha}^{j}(z)=\left(\mu_{\alpha}^{j}\right)^{-\frac{n-2 \gamma}{2}} U_{a_{j}}^{\lambda_{j}}\left(\left(\mu_{\alpha}^{j}\right)^{-1} \varphi_{x_{\alpha}^{j}}^{-1}(z)\right),
$$

for $z \in \varphi_{x_{\alpha}^{j}}\left(B_{r_{0}}^{+}(0)\right)$, and $\varphi_{x_{\alpha}^{j}}$ are Fermi coordinates centered at $x_{\alpha}^{j} \in M$ with $r_{0}>0$ small, and $\eta_{\alpha}^{j}$ are cutoff functions such that

$$
\eta_{\alpha}^{j} \equiv 1 \text { in } \varphi_{x_{\alpha}^{j}}\left(B_{r_{0}}^{+}(0)\right) \quad \text { and } \quad \eta_{\alpha}^{j} \equiv 0 \text { in } M \backslash \varphi_{x_{\alpha}^{j}}\left(B_{2 r_{0}}^{+}(0)\right) \text {; }
$$

(4) The energies

$$
I_{g}^{\gamma, \alpha}\left(u_{\alpha}\right)-I_{g}^{\infty}\left(u^{0}\right)-m \widetilde{E}\left(U_{a_{j}}^{\lambda_{j}}\right) \rightarrow 0, \quad \text { as } \alpha \rightarrow+\infty
$$

(5) For any $1 \leq i, j \leq m, i \neq j$,

$$
\frac{\mu_{\alpha}^{i}}{\mu_{\alpha}^{j}}+\frac{\mu_{\alpha}^{j}}{\mu_{\alpha}^{i}}+\frac{d \hat{h}\left(x_{\alpha}^{i}, x_{\alpha}^{j}\right)^{2}}{\mu_{\alpha}^{i} \mu_{\alpha}^{j}} \rightarrow+\infty, \quad \text { as } \alpha \rightarrow+\infty \text {. }
$$

Remark 1.4. (i) We call $\eta_{\alpha}^{j} u_{\alpha}^{j}$ a bubble for $j=1, \ldots, m$.

(ii) If $u_{\alpha} \rightarrow u^{0}$ strongly in $W^{1,2}\left(X, \rho^{1-2 \gamma}\right)$ as $\alpha \rightarrow+\infty$, then $m=0$.

Although the local case $\gamma=1$ is well known [Druet et al. 2004; Struwe 1984], the most interesting point in the fractional case is the fact that one still has an energy decomposition into bubbles, and that these bubbles are noninterfering, which is surprising since our operator is nonlocal.

We finally recall that in the flat case, compactness problems for the fractional Laplacian were considered in the nice papers by Palatucci and Pisante [2014; 2015], and also the paper by Yan, Yang, and Yu [Yan et al. 2015].

This paper is organized as follows: In Section 2, we will first recall the definition of weighted Sobolev spaces and Palais-Smale sequences. Then we will derive a criterion for the strong convergence of a given Palais-Smale sequence. At last, $\varepsilon$-regularity estimates will be established. In Section 3, we will extract the first bubble from the Palais-Smale sequence which is not strongly convergent. In Section 4, we will give the proof of Theorem 1.3. Finally, some regularity estimates of the degenerate elliptic PDE are given in the Appendix. 


\section{Preliminary results}

Most of the arguments in this section are analogous to the results in [Druet et al. 2004, Chapter 3]. For the convenience to the reader, we also prove these lemmas with the necessary modifications.

From now on we use $2^{*}=2 n /(n-2 \gamma), \gamma \in(0,1)$ for simplicity, and always assume that Palais-Smale sequences are all nonnegative. Moreover, the notation $o(1)$ will be taken with respect to the limit $\alpha \rightarrow+\infty$.

Definition 2.1. The weighted Sobolev space $W^{1,2}\left(X, \rho^{1-2 \gamma}\right)$ is defined as the closure of $\mathscr{C}^{\infty}(\bar{X})$ with norm

$$
\|u\|_{W^{1,2}\left(X, \rho^{1-2 \gamma}\right)}=\left(\int_{X} \rho^{1-2 \gamma}|\nabla u|_{g}^{2} d v_{g}+\int_{M} u^{2} d \sigma_{\hat{h}}\right)^{\frac{1}{2}}
$$

where $d v_{g}$ is the volume form of the asymptotically hyperbolic Riemannian manifold $(X, g)$ and $d \sigma_{\hat{h}}$ is the volume form of the conformal infinity $(M,[\hat{h}])$.

Proposition 2.2. The norm defined above is equivalent to the following traditional norm

$$
\|u\|_{W^{1,2}\left(X, \rho^{1-2 \gamma}\right)}^{*}=\left(\int_{X} \rho^{1-2 \gamma}\left(|\nabla u|_{g}^{2}+u^{2}\right) d v g\right)^{\frac{1}{2}} .
$$

On one hand, $\|\cdot\|$ can be controlled by $\|\cdot\|^{*}$. This is a easy consequence of the following two propositions. The first one is a trace Sobolev embedding on Euclidean space.

Proposition 2.3 [Jin and Xiong 2013]. For any $u \in \mathscr{C}_{0}^{\infty}\left(\mathbb{R}_{+}^{n+1}\right)$,

$$
\left(\int_{\mathbb{R}^{n}}|u(x, 0)|^{2^{*}} d x\right)^{\frac{2}{2^{*}}} \leq S(n, \gamma) \int_{\mathbb{R}_{+}^{n+1}} y^{1-2 \gamma}|\nabla u(x, y)|^{2} d x d y
$$

where

$$
S(n, \gamma)=\frac{1}{2 \pi^{\gamma}} \frac{\Gamma(\gamma)}{\Gamma(1-\gamma)} \frac{\Gamma\left(\frac{n-2 \gamma}{2}\right)}{\Gamma\left(\frac{n+2 \gamma}{2}\right)}\left(\frac{\Gamma(n)}{\Gamma\left(\frac{n}{2}\right)}\right)^{\frac{2 \gamma}{n}} .
$$

Using a standard partition of unity argument, one obtains a weighted trace Sobolev inequality on an asymptotically hyperbolic manifold:

Proposition 2.4 [Jin and Xiong 2013]. For any $\varepsilon>0$, there exists a constant $C_{\varepsilon}>0$ such that

$$
\left(\int_{M}|u|^{2^{*}} d \sigma_{\hat{h}}\right)^{\frac{2}{2^{*}}} \leq(S(n, \gamma)+\varepsilon) \int_{X} \rho^{1-2 \gamma}|\nabla u|_{g}^{2} d v_{g}+C_{\varepsilon} \int_{X} \rho^{1-2 \gamma} u^{2} d v_{g} .
$$


On the other hand, $\|\cdot\|^{*}$ can be controlled by $\|\cdot\|$, which is implied by the following proposition.

Proposition 2.5. For any $u \in W^{1,2}\left(X, \rho^{1-2 \gamma}\right)$, there exists a constant $C>0$ such that

$$
\int_{X} \rho^{1-2 \gamma} u^{2} d v_{g} \leq C\left(\int_{X} \rho^{1-2 \gamma}|\nabla u|_{g}^{2} d v_{g}+\int_{M} u^{2} d \sigma_{\hat{h}}\right) .
$$

Proof. We use a contradiction argument. Thus, assume that for any $\alpha \geq 1$ there exists $u_{\alpha}$ satisfying

$$
\int_{X} \rho^{1-2 \gamma} u_{\alpha}^{2} d v_{g} \geq \alpha\left(\int_{X} \rho^{1-2 \gamma}\left|\nabla u_{\alpha}\right|_{g}^{2} d v_{g}+\int_{M} u_{\alpha}^{2} d \sigma_{\hat{h}}\right) .
$$

Without loss of generality, we can assume that $\int_{X} \rho^{1-2 \gamma} u_{\alpha}^{2} d v_{g}=1$. Then we have

$$
\int_{X} \rho^{1-2 \gamma}\left(\left|\nabla u_{\alpha}\right|_{g}^{2}+u_{\alpha}^{2}\right) d v_{g} \leq 1+\frac{1}{\alpha}
$$

Then there exists a weakly convergent subsequence, also denoted by $\left\{u_{\alpha}\right\}$, such that $u_{\alpha} \rightarrow u_{0}$ in $W^{1,2}\left(X, \rho^{1-2 \gamma},\|\cdot\|^{*}\right)$.

Since

$$
\lim _{\alpha \rightarrow \infty} \int_{X} \rho^{1-2 \gamma}\left|\nabla u_{\alpha}\right|_{g}^{2} d v_{g}=0 \quad \text { and } \quad \lim _{\alpha \rightarrow \infty} \int_{M} u_{\alpha}^{2} d \sigma_{\hat{h}}=0,
$$

we get that $u_{0} \equiv 0$. On the other hand, via the following Proposition 2.6, the embedding $W^{1,2}\left(X, \rho^{1-2 \gamma},\|\cdot\|^{*}\right) \hookrightarrow L^{2}\left(X, \rho^{1-2 \gamma}\right)$ is compact. So we have

$$
\int_{X} \rho^{1-2 \gamma} u_{0}^{2} d v_{g}=1
$$

which contradicts the fact that $u_{0} \equiv 0$. Then the proof is completed.

Proposition 2.6 [Jin and Xiong 2013; Kufner 1985; Di Nezza et al. 2012]. Let $1 \leq p \leq q<\infty$ with $\frac{1}{n+1}>\frac{1}{p}-\frac{1}{q}$.

(i) Suppose $2-2 \gamma \leq p$. Then $W^{1, p}\left(X, \rho^{1-2 \gamma},\|\cdot\|^{*}\right)$ is compactly embedded in $L^{q}\left(X, \rho^{1-2 \gamma}\right)$ if

$$
\frac{2-2 \gamma}{p(n+2-2 \gamma)}>\frac{1}{p}-\frac{1}{q}
$$

(ii) Suppose $2-2 \gamma>p$. Then $W^{1, p}\left(X, \rho^{1-2 \gamma},\|\cdot\|^{*}\right)$ is compactly embedded in $L^{q}\left(X, \rho^{1-2 \gamma}\right)$ if and only if

$$
\frac{1}{(n+2-2 \gamma)}>\frac{1}{p}-\frac{1}{q} \text {. }
$$

We will always use the norm in $W^{1,2}\left(X, \rho^{1-2 \gamma}\right)$ in the following unless otherwise stated. 
Definition 2.7. The weighted Sobolev space $\bar{W}^{1,2}\left(X, \rho^{1-2 \gamma}\right)$ is the closure of $\mathscr{C}_{0}^{\infty}(X)$ in $W^{1,2}\left(X, \rho^{1-2 \gamma}\right)$ with the norm

$$
\|u\|_{\bar{W}^{1,2}\left(X, \rho^{1-2 \gamma}\right)}=\left(\int_{X} \rho^{1-2 \gamma}|\nabla u|_{g}^{2} d v_{g}\right)^{\frac{1}{2}} .
$$

Now we define Palais-Smale sequences for the functional (1-11) precisely.

Definition 2.8. The sequence $\left\{u_{\alpha}\right\}_{\alpha \in \mathbb{N}} \subset W^{1,2}\left(X, \rho^{1-2 \gamma}\right)$ is called a Palais-Smale sequence for $\left\{I_{g}^{\gamma, \alpha}\right\}_{\alpha \in \mathbb{N}}$ if:

(i) $\left\{I_{g}^{\gamma, \alpha}\left(u_{\alpha}\right)\right\}_{\alpha \in \mathbb{N}}$ is uniformly bounded; and

(ii) as $\alpha \rightarrow+\infty$,

$$
D I_{g}^{\gamma, \alpha}\left(u_{\alpha}\right) \rightarrow 0, \text { strongly in } W^{1,2}\left(X, \rho^{1-2 \gamma}\right)^{\prime},
$$

where we have defined $W^{1,2}\left(X, \rho^{1-2 \gamma}\right)^{\prime}$ as the dual space of $W^{1,2}\left(X, \rho^{2 \gamma-1}\right)$, i.e., for any $\theta \in W^{1,2}\left(X, \rho^{1-2 \gamma}\right)$,

$$
\begin{aligned}
& D I_{g}^{\gamma, \alpha}\left(u_{\alpha}\right) \cdot \theta \\
& \quad=\int_{X} \rho^{1-2 \gamma}\left\langle\nabla u_{\alpha}, \nabla \theta\right\rangle_{g} d v_{g}+\int_{M} Q_{\alpha}^{\gamma} u_{\alpha} \theta d \sigma_{\hat{h}}-\int_{M} u_{\alpha}^{2^{*}-1} \theta d \sigma_{\hat{h}} \\
& \quad=o\left(\|\theta\|_{W^{1,2}\left(X, \rho^{1-2 \gamma}\right)}\right), \quad \text { as } \alpha \rightarrow+\infty .
\end{aligned}
$$

The main properties of Palais-Smale sequences are contained in the next several lemmas:

Lemma 2.9. Let $\left\{u_{\alpha}\right\}_{\alpha \in \mathbb{N}} \subset W^{1,2}\left(X, \rho^{1-2 \gamma}\right)$ be a Palais-Smale sequence for the functionals $\left\{I_{g}^{\gamma, \alpha}\right\}_{\alpha \in \mathbb{N}}$, then $\left\{u_{\alpha}\right\}_{\alpha \in \mathbb{N}}$ is uniformly bounded in $W^{1,2}\left(X, \rho^{1-2 \gamma}\right)$.

Proof. We can take $\theta=u_{\alpha} \in W^{1,2}\left(X, \rho^{1-2 \gamma}\right)$ as a test function in (ii) of Definition 2.8. Then, we get

$$
\int_{X} \rho^{1-2 \gamma}\left|\nabla u_{\alpha}\right|_{g}^{2} d v_{g}+\int_{M} Q_{\alpha}^{\gamma} u_{\alpha}^{2} d \sigma_{\hat{h}}=\int_{M} u_{\alpha}^{2^{*}} d \sigma_{\hat{h}}+o\left(\left\|u_{\alpha}\right\|_{W^{1,2}\left(X, \rho^{1-2 \gamma}\right)}\right),
$$

which yields that

$$
\begin{aligned}
I_{g}^{\gamma, \alpha}\left(u_{\alpha}\right) & =\frac{1}{2} \int_{X} \rho^{1-2 \gamma}\left|\nabla u_{\alpha}\right|_{g}^{2} d v_{g}+\frac{1}{2} \int_{M} Q_{\alpha}^{\gamma} u_{\alpha}^{2} d \sigma_{\hat{h}}-\frac{1}{2} * \int_{M} u_{\alpha}^{2^{*}} d \sigma_{\hat{h}} \\
& =\frac{\gamma}{n} \int_{M} u_{\alpha}^{2^{*}} d \sigma_{\hat{h}}+o\left(\left\|u_{\alpha}\right\|_{W^{1,2}\left(X, \rho^{1-2 \gamma}\right)}\right) .
\end{aligned}
$$

Since $\left\{I_{g}^{\gamma, \alpha}\left(u_{\alpha}\right)\right\}_{\alpha \in \mathbb{N}}$ is uniformly bounded by (i) of Definition 2.8, there exists a constant $C>0$ such that

$$
\int_{M} u_{\alpha}^{2^{*}} d \sigma_{\hat{h}} \leq C+o\left(\left\|u_{\alpha}\right\|_{W^{1,2}\left(X, \rho^{1-2 \gamma}\right)}\right),
$$


which by Hölder's inequality yields

$$
\int_{M} u_{\alpha}^{2} d \sigma_{\hat{h}} \leq C\left(\int_{M} u_{\alpha}^{2^{*}} d \sigma_{\hat{h}}\right)^{\frac{2}{2^{*}}} \leq C+o\left(\left\|u_{\alpha}\right\|_{W^{1,2}\left(X, \rho^{1-2 \gamma}\right)}^{2 / 2^{*}}\right) .
$$

Note that since $\left|Q_{\alpha}^{\gamma}\right| \leq C$ for some constant $C>0$, we can choose sufficiently large $C_{1}>0$ such that $C_{1}+Q_{\alpha}^{\gamma} \geq 1$ on $M$. It follows that

$$
\begin{aligned}
\left\|u_{\alpha}\right\|_{W^{1,2}\left(X, \rho^{1-2 \gamma}\right)}^{2} & =\int_{X} \rho^{1-2 \gamma}\left|\nabla u_{\alpha}\right|_{g}^{2} d v_{g}+\int_{M} u_{\alpha}^{2} d \sigma_{\hat{h}} \\
& \leq \int_{X} \rho^{1-2 \gamma}\left|\nabla u_{\alpha}\right|_{g}^{2} d v_{g}+\int_{M} Q_{\alpha}^{\gamma} u_{\alpha}^{2} d \sigma_{\hat{h}}+C_{1} \int_{M} u_{\alpha}^{2} d \sigma_{\hat{h}} \\
& \leq \int_{M} u_{\alpha}^{2^{*}} d \sigma_{\hat{h}}+o\left(\left\|u_{\alpha}\right\|_{W^{1,2}\left(X, \rho^{1-2 \gamma}\right)}\right)+C+o\left(\left\|u_{\alpha}\right\|_{W^{1,2}\left(X, \rho^{1-2 \gamma)}\right)}^{22^{*}}\right) \\
& \leq C+o\left(\left\|u_{\alpha}\right\|_{W^{1,2}\left(X, \rho^{1-2 \gamma}\right)}\right)+o\left(\left\|u_{\alpha}\right\|_{W^{1,2}\left(X, \rho^{1-2 \gamma}\right)}^{2 / 2^{*}}\right),
\end{aligned}
$$

from which we conclude that $\left\{u_{\alpha}\right\}_{\alpha \in \mathbb{N}}$ is uniformly bounded in $W^{1,2}\left(X, \rho^{1-2 \gamma}\right)$ since $2 / 2^{*}<1$.

Remark 2.10. From Lemma 2.9 , it is easy to see that there exists a function $u^{0}$ in $W^{1,2}\left(X, \rho^{1-2 \gamma}\right)$ such that $u_{\alpha} \rightarrow u^{0}$ weakly in $W^{1,2}\left(X, \rho^{1-2 \gamma}\right)$ as $\alpha \rightarrow+\infty$.

Proposition 2.11. The function $u^{0}$ is nonnegative in $\bar{X}$.

Proof. Using Proposition 2.4, we can easily get that $u_{\alpha} \rightarrow u^{0}$ in $L^{2}(M, \hat{h})$ as $\alpha \rightarrow+\infty$, so we have $u_{\alpha} \rightarrow u^{0}$ almost everywhere on $M$. Noting that $u_{\alpha} \geq 0$ on $M$, we obtain that $u^{0} \geq 0$ on $M$. On the other hand, by Proposition 2.6 and by the equivalence of the norms $\|\cdot\|$ and $\|\cdot\|^{*}$, we have $u_{\alpha} \rightarrow u^{0}$ in $L^{2}\left(X, \rho^{1-2 \gamma}\right)$ as $\alpha \rightarrow+\infty$. For any $z \in X$, take $d_{z}<\operatorname{dist}(z, M)$; then we also have $u_{\alpha} \rightarrow u^{0}$ in $L^{2}\left(\mathfrak{B}_{d_{z}}^{+}(z), \rho^{1-2 \gamma}\right)$. Since $\rho^{1-2 \gamma}$ is bounded below by a positive constant in $\mathfrak{B}_{d_{z}}^{+}(z)$, we get $u_{\alpha} \rightarrow u^{0}$ almost everywhere in $\mathfrak{B}_{d_{z}}^{+}(z)$, up to passing to a subsequence. Noting that $u_{\alpha} \geq 0$ in $X$, we obtain $u^{0} \geq 0$ in $\mathfrak{B}_{d_{z}}^{+}(z)$. Since $z$ is arbitrary in $X$, we have $u^{0} \geq 0$ in $X$. Combining the above arguments, we conclude that $u \geq 0$ in $\bar{X}$.

Next we define the two limit functionals

$$
I_{g}^{\gamma}(u)=\frac{1}{2} \int_{X} \rho^{1-2 \gamma}|\nabla u|_{g}^{2} d v_{g}-\frac{1}{2^{*}} \int_{M}|u|^{2^{*}} d \sigma_{\hat{h}}
$$

and

$$
I_{g}^{\gamma, \infty}(u)=\frac{1}{2} \int_{X} \rho^{1-2 \gamma}|\nabla u|_{g}^{2} d v_{g}+\frac{1}{2} \int_{M} Q_{\infty}^{\gamma} u^{2} d \sigma_{\hat{h}}-\frac{1}{2^{*}} \int_{M}|u|^{2^{*}} d \sigma_{\hat{h}} .
$$


Lemma 2.12. Let $\left\{u_{\alpha}\right\}_{\alpha \in \mathbb{N}} \subset W^{1,2}\left(X, \rho^{1-2 \gamma}\right)$ be a Palais-Smale sequence for $\left\{I_{g}^{\gamma, \alpha}\right\}_{\alpha \in \mathbb{N}}$, and $u_{\alpha} \rightarrow u^{0}$ weakly in $W^{1,2}\left(X, \rho^{1-2 \gamma}\right)$ as $\alpha \rightarrow+\infty$. We also set $\hat{u}_{\alpha}=u_{\alpha}-u^{0} \in W^{1,2}\left(X, \rho^{1-2 \gamma}\right)$. Then,

(i) $u^{0}$ is a nonnegative weak solution to the limit equation

$$
\begin{cases}-\operatorname{div}\left(\rho^{1-2 \gamma} \nabla u\right)=0 & \text { in } X, \\ -\lim _{\rho \rightarrow 0} \rho^{1-2 \gamma} \partial_{\rho} u+Q_{\infty}^{\gamma} u=u^{2^{*}-1} & \text { on } M ;\end{cases}
$$

(ii) $I_{g}^{\gamma, \alpha}\left(u_{\alpha}\right)=I_{g}^{\gamma}\left(\hat{u}_{\alpha}\right)+I_{g}^{\gamma, \infty}\left(u^{0}\right)+o(1)$ as $\alpha \rightarrow+\infty$;

(iii) $\left\{\hat{u}_{\alpha}\right\}_{\alpha \in \mathbb{N}}$ is a Palais-Smale sequence for $I_{g}^{\gamma}$.

Proof. (i) As $\mathscr{C}^{\infty}(\bar{X})$ is dense in $W^{1,2}\left(X, \rho^{1-2 \gamma}\right)$, we only consider the proof in $\mathscr{C}^{\infty}(\bar{X})$. Let $\theta \in \mathscr{C}^{\infty}(\bar{X})$. Since $Q_{\alpha}^{\gamma} \rightarrow Q_{\infty}^{\gamma}$ in $L^{2}(M, \hat{h})$ as $\alpha \rightarrow+\infty$ and $u_{\alpha} \rightarrow u^{0}$ weakly in $W^{1,2}\left(X, \rho^{1-2 \gamma}\right)$ as $\alpha \rightarrow+\infty$,

$$
\int_{M} Q_{\alpha}^{\gamma} u_{\alpha} \theta d \sigma_{\hat{h}}=\int_{M} Q_{\infty}^{\gamma} u^{0} \theta d \sigma_{\hat{h}}+o(1)
$$

Passing to the limit in (2-3), we get easily that

$$
\int_{X} \rho^{1-2 \gamma}\left\langle\nabla u^{0}, \nabla \theta\right\rangle_{g} d v_{g}+\int_{M} Q_{\infty}^{\gamma} u^{0} \theta d \sigma_{\hat{h}}=\int_{M}\left(u^{0}\right)^{2^{*}-1} \theta d \sigma_{\hat{h}},
$$

i.e., $u^{0}$ is a weak solution to the limit equation (2-4).

For the proof of (ii), recall that

$$
\int_{M} Q_{\alpha}^{\gamma} u_{\alpha}^{2} d \sigma_{\hat{h}}=\int_{M} Q_{\infty}^{\gamma}\left(u^{0}\right)^{2} d \sigma_{\hat{h}}+o(1)
$$

and

$$
\begin{aligned}
I_{g}^{\gamma, \alpha}\left(u_{\alpha}\right) & =\frac{1}{2} \int_{X} \rho^{1-2 \gamma}\left|\nabla u_{\alpha}\right|_{g}^{2} d v_{g}+\frac{1}{2} \int_{M} Q_{\alpha}^{\gamma} u_{\alpha}^{2} d \sigma_{\hat{h}}-\frac{1}{2^{*}} \int_{M} u_{\alpha}^{2^{*}} d \sigma_{\hat{h}}, \\
I_{g}^{\gamma, \infty}\left(u^{0}\right) & =\frac{1}{2} \int_{X} \rho^{1-2 \gamma}\left|\nabla u^{0}\right|_{g}^{2} d v_{g}+\frac{1}{2} \int_{M} Q_{\infty}^{\gamma}\left(u^{0}\right)^{2} d \sigma_{\hat{h}}-\frac{1}{2^{*}} \int_{M}\left(u^{0}\right)^{2^{*}} d \sigma_{\hat{h}}, \\
I_{g}^{\gamma}\left(\hat{u}_{\alpha}\right) & =\frac{1}{2} \int_{X} \rho^{1-2 \gamma}\left|\nabla \hat{u}_{\alpha}\right|_{g}^{2} d v_{g}-\frac{1}{2^{*}} \int_{M}\left|\hat{u}_{\alpha}\right|^{2^{*}} d \sigma_{\hat{h}},
\end{aligned}
$$

where $\hat{u}_{\alpha}=u_{\alpha}-u^{0}$. Then,

$$
\begin{aligned}
I_{g}^{\gamma, \alpha}\left(u_{\alpha}\right)-I_{g}^{\gamma, \infty}\left(u^{0}\right)-I_{g}^{\gamma} & \left(\hat{u}_{\alpha}\right) \\
& =\int_{X} \rho^{1-2 \gamma}\left\langle\nabla u^{0}, \nabla \hat{u}_{\alpha}\right\rangle_{g} d v_{g}-\frac{1}{2^{*}} \int_{M} \Phi_{\alpha} d \sigma_{\hat{h}}+o(1),
\end{aligned}
$$

where

$$
\Phi_{\alpha}=\left|\hat{u}_{\alpha}+u^{0}\right|^{2^{*}}-\left|\hat{u}_{\alpha}\right|^{2^{*}}-\left|u^{0}\right|^{2^{*}}
$$


Note that $\hat{u}_{\alpha} \rightarrow 0$ weakly in $W^{1,2}\left(X, \rho^{1-2 \gamma}\right)$ as $\alpha \rightarrow+\infty$, thus

$$
\int_{X} \rho^{1-2 \gamma}\left\langle\nabla u^{0}, \nabla \hat{u}_{\alpha}\right\rangle_{g} d v_{g} \rightarrow 0, \quad \text { as } \alpha \rightarrow \infty .
$$

On the other hand, it is easy to check that there exists a constant $C>0$, independent of $\alpha$, such that

$$
|| \hat{u}_{\alpha}+\left.u^{0}\right|^{2^{*}}-\left|\hat{u}_{\alpha}\right|^{2^{*}}-\left|u^{0}\right|^{2^{*}} \mid \leq C\left(\left|\hat{u}_{\alpha}\right|^{2^{*}-1}\left|u^{0}\right|+\left|u^{0}\right|^{2^{*}-1}\left|\hat{u}_{\alpha}\right|\right) .
$$

As a consequence, since $\hat{u}_{\alpha} \rightarrow 0$ weakly in $L^{2^{*}}(M, \hat{h})$ by Proposition 2.4 , we have

$$
\int_{M}\left|\Phi_{\alpha}\right| d \sigma_{\hat{h}} \rightarrow 0, \quad \text { as } \alpha \rightarrow+\infty \text {. }
$$

The proof of (ii) is completed.

(iii) For any $\theta \in \mathscr{C}^{\infty}(\bar{X})$, by (i) we have

$$
D I_{g}^{\gamma, \infty}\left(u^{0}\right) \cdot \theta=0 .
$$

Since, in addition,

$$
\int_{M} Q_{\alpha}^{\gamma} u_{\alpha} \theta d \sigma_{\hat{h}}=\int_{M} Q_{\infty}^{\gamma} u^{0} \theta d \sigma_{\hat{h}}+o\left(\|\theta\|_{W^{1,2}\left(X, \rho^{1-2 \gamma}\right)}\right),
$$

then

$$
D I_{g}^{\gamma, \alpha}\left(u_{\alpha}\right) \cdot \theta=D I_{g}^{\gamma}\left(\hat{u}_{\alpha}\right) \cdot \theta-\int_{M} \Psi_{\alpha} \theta d \sigma_{\hat{h}}+o\left(\|\theta\|_{W^{1,2}\left(X, \rho^{1-2 \gamma}\right)}\right),
$$

where $\Psi_{\alpha}=\left|\hat{u}_{\alpha}+u^{0}\right|^{2^{*}-2}\left(\hat{u}_{\alpha}+u^{0}\right)-\left|\hat{u}_{\alpha}\right|^{2^{*}-2} \hat{u}_{\alpha}-\left|u^{0}\right|^{2^{*}-2} u^{0}$, and it is easy to check that there exists a constant $C>0$ independent of $\alpha$ such that

$$
\left|\Psi_{\alpha}\right| \leq C\left(\left|\hat{u}_{\alpha}\right|^{2^{*}-2}\left|u^{0}\right|+\left|\hat{u}_{\alpha}\right|\left|u^{0}\right|^{2^{*}-2}\right) .
$$

By Hölder's inequality and the fact $\hat{u}_{\alpha} \rightarrow 0$ weakly in $W^{1,2}\left(X, \rho^{1-2 \gamma}\right)$ as $\alpha \rightarrow+\infty$,

$$
\begin{aligned}
& \int_{M} \Psi_{\alpha} \theta d \sigma_{\hat{h}} \\
& \quad \leq\left(\left\|\left|\hat{u}_{\alpha}\right|^{2^{*}-2}\left|u^{0}\right|\right\|_{L^{2^{*} /\left(2^{*}-1\right)}(M)}+\left\|\left|\hat{u}_{\alpha}\right|\left|u^{0}\right|^{2^{*}-2}\right\|_{L^{2^{*} /\left(2^{*}-1\right)}(M)}\right)\|\theta\|_{L^{2^{*}(M)}} \\
& \quad=o(1)\|\theta\|_{L^{2^{*}}(M)} .
\end{aligned}
$$

Thus from (2-5),

$$
D I_{g}^{\gamma, \alpha}\left(u_{\alpha}\right) \cdot \theta=D I_{g}^{\gamma}\left(\hat{u}_{\alpha}\right) \cdot \theta+o(1)\|\theta\|_{L^{2 *}(M)},
$$

which implies that $D I_{g}^{\gamma}\left(\hat{u}_{\alpha}\right) \rightarrow 0$ in $W^{1,2}\left(X, \rho^{1-2 \gamma}\right)^{\prime}$ as $\alpha \rightarrow+\infty$, since $\left\{u_{\alpha}\right\}_{\alpha \in \mathbb{N}}$ is a Palais-Smale sequence for $\left\{I_{g}^{\gamma, \alpha}\right\}_{\alpha \in \mathbb{N}}$. 
Finally, from (ii), we know that $\left\{\hat{u}_{\alpha}\right\}_{\alpha \in \mathbb{N}}$ is a Palais-Smale sequence for $I_{g}^{\gamma}$. This completes the proof of the lemma.

Now we give a criterion for strong convergence of Palais-Smale sequences.

Lemma 2.13. Let $\left\{\hat{u}_{\alpha}\right\}_{\alpha \in \mathbb{N}}$ be a Palais-Smale sequence for $I_{g}^{\gamma}$ such that $\hat{u}_{\alpha} \rightarrow 0$ weakly in $W^{1,2}\left(X, \rho^{1-2 \gamma}\right)$ as $\alpha \rightarrow+\infty$. If $I_{g}^{\gamma}\left(\hat{u}_{\alpha}\right) \rightarrow \beta$ and

$$
\beta<\beta_{0}=\frac{\gamma}{n}\left(d_{\gamma}^{*}\right)^{-\frac{n}{2 \gamma}} \Lambda_{\gamma}(M,[\hat{h}])^{\frac{n}{2 \gamma}},
$$

then $\hat{u}_{\alpha} \rightarrow 0$ in $W^{1,2}\left(X, \rho^{1-2 \gamma}\right)$ as $\alpha \rightarrow+\infty$.

Proof. By Lemma 2.9 (here $Q_{\alpha}^{\gamma} \equiv 0$ ), there exists a constant $C>0$ such that $\left\|\hat{u}_{\alpha}\right\|_{W^{1,2}\left(X, \rho^{1-2 \gamma}\right)} \leq C$ for all $\alpha \in \mathbb{N}$, so

$$
\begin{aligned}
D I_{g}^{\gamma}\left(\hat{u}_{\alpha}\right) \cdot \hat{u}_{\alpha} & =\int_{X} \rho^{1-2 \gamma}\left|\nabla \hat{u}_{\alpha}\right|_{g}^{2} d v_{g}-\int_{M}\left|\hat{u}_{\alpha}\right|^{2^{*}} d \sigma_{\hat{h}} \\
& =o\left(\left\|\hat{u}_{\alpha}\right\|_{W^{1,2}\left(X, \rho^{1-2 \gamma}\right)}\right)=o(1) .
\end{aligned}
$$

Then note that $I_{g}^{\gamma}\left(\hat{u}_{\alpha}\right) \rightarrow \beta$ as $\alpha \rightarrow+\infty$, so

$$
\begin{aligned}
\beta+o(1) & =I_{g}^{\gamma}\left(\hat{u}_{\alpha}\right) \\
& =\frac{1}{2} \int_{X} \rho^{1-2 \gamma}\left|\nabla \hat{u}_{\alpha}\right|_{g}^{2} d v_{g}-\frac{1}{2^{*}} \int_{M}\left|\hat{u}_{\alpha}\right|^{2^{*}} d \sigma_{\hat{h}} \\
& =\frac{\gamma}{n} \int_{X} \rho^{1-2 \gamma}\left|\nabla \hat{u}_{\alpha}\right|_{g}^{2} d v_{g}+o(1) \\
& =\frac{\gamma}{n} \int_{M}\left|\hat{u}_{\alpha}\right|^{2^{*}} d \sigma_{\hat{h}}+o(1) .
\end{aligned}
$$

On the other hand, in the positive curvature case, it was shown in [González and Qing 2013] that the $\gamma$-Yamabe constant (1-9) must be positive: $\Lambda_{\gamma}(M,[\hat{h}])>0$. Moreover, by definition,

$$
\begin{aligned}
\Lambda_{\gamma}(M,[\hat{h}])\left(\int_{M}\left|\hat{u}_{\alpha}\right|^{2^{*}} d \sigma_{\hat{h}}\right)^{\frac{2}{2^{*}}} & \\
& \leq d_{\gamma}^{*} \int_{X} \rho^{1-2 \gamma}\left|\nabla \hat{u}_{\alpha}\right|_{g}^{2} d v_{g}+\int_{M} Q_{\gamma}^{\hat{h}} \hat{u}_{\alpha}^{2} d \sigma_{\hat{h}} .
\end{aligned}
$$

where $d_{\gamma}^{*}>0$. We also know that $\left|Q_{\gamma}^{\hat{h}}\right| \leq C$ on $M^{n}$. Note that, by Proposition 2.4, $\hat{u}_{\alpha} \rightarrow 0$ in $L^{2^{*}}(M, \hat{h})$ as $\alpha \rightarrow+\infty$, so

$$
\int_{M} \hat{u}_{\alpha}^{2} d \sigma_{\hat{h}} \rightarrow 0, \quad \text { as } \alpha \rightarrow+\infty
$$


since the embedding $L^{2^{*}}(M, \hat{h}) \subset L^{2}(M, \hat{h})$ is compact. So we get from (2-7) and (2-8) that

$$
\left(\frac{n}{\gamma} \beta+o(1)\right)^{\frac{2}{2^{*}}} \leq d_{\gamma}^{*} \Lambda_{\gamma}(M,[\hat{h}])^{-1} \frac{n}{\gamma} \beta+o(1) .
$$

Taking $\alpha \rightarrow+\infty$, we must have $\beta=0$ because of our initial condition (2-6).

Note that the Palais-Smale condition (ii) is the weak form of a Dirichlet-toNeumann problem for a degenerate elliptic PDE. In fact, as $D I_{g}^{\gamma}\left(\hat{u}_{\alpha}\right) \rightarrow 0$ in $W^{1,2}\left(X, \rho^{1-2 \gamma}\right)^{\prime}$, it follows that, for any $\psi \in W^{1,2}\left(X, \rho^{1-2 \gamma}\right)$,

$$
\begin{aligned}
\int_{X} \rho^{1-2 \gamma}\left\langle\nabla \hat{u}_{\alpha}, \nabla \psi\right\rangle_{g} d v_{g}-\int_{M}\left|\hat{u}_{\alpha}\right|^{2^{*}-2} \hat{u}_{\alpha} \psi & d \sigma_{\hat{h}} \\
& =o(1)\|\psi\|_{W^{1,2}\left(X, \rho^{1-2 \gamma}\right)} .
\end{aligned}
$$

In particular, for any $\bar{\psi} \in \bar{W}^{1,2}\left(X, \rho^{1-2 \gamma}\right)$,

$$
\int_{X} \rho^{1-2 \gamma}\left\langle\nabla \hat{u}_{\alpha}, \nabla \bar{\psi}\right\rangle_{g} d v_{g}=o(1)\|\bar{\psi}\|_{\bar{W}^{1,2}\left(X, \rho^{1-2 \gamma}\right)},
$$

which is precisely the weak formulation of the asymptotic equation

$$
-\operatorname{div}\left(\rho^{1-2 \gamma} \nabla \hat{u}_{\alpha}\right)=o(1) \quad \text { in } X .
$$

Multiplying both sides of (2-10) by $\psi \in W^{1,2}\left(X, \rho^{1-2 \gamma}\right)$ and integrating by parts, we obtain

$\int_{M} \lim _{\rho \rightarrow 0} \rho^{1-2 \gamma} \partial_{\rho} \hat{u}_{\alpha} \psi d \sigma_{\hat{h}}+\int_{X} \rho^{1-2 \gamma}\left\langle\nabla \hat{u}_{\alpha}, \nabla \psi\right\rangle_{g} d v_{g}=o(1)\|\psi\|_{W^{1,2}\left(X, \rho^{1-2 \gamma}\right)}$, which, combined with (2-9), yields that

$$
\int_{M} \lim _{\rho \rightarrow 0} \rho^{1-2 \gamma} \partial_{\rho} \hat{u}_{\alpha} \psi d \sigma_{\hat{h}}+\int_{M}\left|\hat{u}_{\alpha}\right|^{2^{*}-2} \hat{u}_{\alpha} \psi d \sigma_{\hat{h}}=o(1)\|\psi\|_{W^{1,2}\left(X, \rho^{1-2 \gamma}\right)},
$$

and this is precisely the boundary equation in the weak sense

$$
-\lim _{\rho \rightarrow 0} \rho^{1-2 \gamma} \partial_{\rho} \hat{u}_{\alpha}=\left|\hat{u}_{\alpha}\right|^{2^{*}-2} \hat{u}_{\alpha}+o(1) \quad \text { on } M .
$$

For (2-10) and (2-11) with $\left\{\hat{u}_{\alpha}\right\}_{\alpha \in \mathbb{N}}$, we have the following energy estimate, which will play an important role in the proof of the strong convergence in Section 3 . We use the notation $\mathfrak{B}_{r}^{+}$instead of $\mathfrak{B}_{r}^{+}(0)$ for convenience.

Lemma 2.14. (E-regularity estimates) Suppose that $\left\{v_{\alpha}\right\}_{\alpha \in \mathbb{N}}$ satisfies the following asymptotic boundary value problem

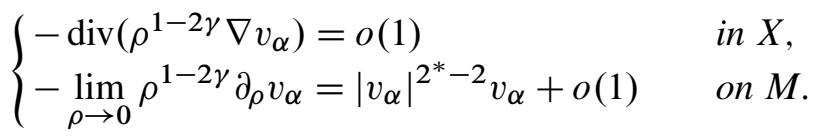


If there exists small $\varepsilon>0$ depending on $n$ and $\gamma$ such that $\int_{\partial^{\prime} \mathfrak{B}_{2 r}^{+}}\left|v_{\alpha}\right|^{2^{*}} d \sigma_{\hat{h}} \leq \varepsilon$
uniformly in $\alpha$ for some small $r>0$, then

$$
\begin{array}{rl}
\int_{\mathfrak{B}_{r}^{+}} \rho^{1-2 \gamma}\left|\nabla v_{\alpha}\right|_{g}^{2} & d v_{g} \\
\leq & \frac{C}{r^{2}} \int_{\mathfrak{B}_{2 r}^{+}} \rho^{1-2 \gamma} v_{\alpha}^{2} d v_{g}+C \int_{\partial^{\prime} \mathfrak{B}_{2 r}^{+}} v_{\alpha}^{2} d \sigma_{\hat{h}}+o(1) \int_{\mathfrak{B}_{2 r}^{+}}\left|v_{\alpha}\right| d v_{g},
\end{array}
$$

where $C=C(n, \varepsilon, \gamma)$ independent of $\alpha$.

Proof. Let $\eta$ be a smooth cutoff function in $\bar{X}$ such that $0 \leq \eta \leq 1, \eta \equiv 1$ in $\mathfrak{B}_{r}^{+}(0)$ and $\eta \equiv 0$ in $\bar{X} \backslash \mathfrak{B}_{2 r}^{+}(0)$. Multiplying both sides of the first equation in (2-12) by $\eta^{2} v_{\alpha}$, integrating by parts and substituting the second equation in (2-12), we get

$$
\begin{aligned}
\int_{\mathfrak{B}_{2 r}^{+}} \rho^{1-2 \gamma}\left\langle\nabla v_{\alpha}, \nabla\right. & \left.\left(\eta^{2} v_{\alpha}\right)\right\rangle_{g} d v_{g} \\
& =-\int_{\partial^{\prime} \mathfrak{B}_{2 r}^{+}} \lim _{\rho \rightarrow 0} \rho^{1-2 \gamma}\left(\partial_{\rho} v_{\alpha}\right) \eta^{2} v_{\alpha} d \sigma_{\hat{h}}+o(1) \int_{\mathfrak{B}_{2 r}^{+}} \eta^{2} v_{\alpha} d v_{g} \\
& =\int_{\partial^{\prime} \mathfrak{B}_{2 r}^{+}} \eta^{2}\left|v_{\alpha}\right|^{2^{*}} d \sigma_{\hat{h}}+o(1) \int_{\mathfrak{B}_{2 r}^{+}} \eta^{2} v_{\alpha} d v_{g},
\end{aligned}
$$

so we have

$$
\begin{aligned}
\int_{\mathfrak{B}_{2 r}^{+}} \rho^{1-2 \gamma} \eta^{2}\left|\nabla v_{\alpha}\right|_{g}^{2} d v_{g} & \\
=- & \int_{\mathfrak{B}_{2 r}^{+}} \rho^{1-2 \gamma} 2 \eta v_{\alpha}\left\langle\nabla v_{\alpha}, \nabla \eta\right\rangle_{g} d v_{g} \\
& +\int_{\partial^{\prime} \mathfrak{B}_{2 r}^{+}} \eta^{2}\left|v_{\alpha}\right|^{2^{*}} d \sigma_{\hat{h}}+o(1) \int_{\mathfrak{B}_{2 r}^{+}} \eta^{2}\left|v_{\alpha}\right| d v_{g} \\
\leq & \frac{1}{2} \int_{\mathfrak{B}_{2 r}^{+}} \eta^{2} \rho^{1-2 \gamma}\left|\nabla v_{\alpha}\right|_{g}^{2} d v_{g}+2 \int_{\mathfrak{B}_{2 r}^{+}} \rho^{1-2 \gamma}|\nabla \eta|_{g}^{2} v_{\alpha}^{2} d v_{g} \\
& +\int_{\partial^{\prime} \mathfrak{B}_{2 r}^{+}} \eta^{2}\left|v_{\alpha}\right|^{2^{*}} d \sigma_{\hat{h}}+o(1) \int_{\mathfrak{B}_{2 r}^{+}} \eta^{2}\left|v_{\alpha}\right| d v_{g},
\end{aligned}
$$

which implies that

$$
\begin{aligned}
& \int_{\mathfrak{B}_{2 r}^{+}} \rho^{1-2 \gamma} \eta^{2}\left|\nabla v_{\alpha}\right|_{g}^{2} d v_{g} \\
& \quad \leq 4 \int_{\mathfrak{B}_{2 r}^{+}} \rho^{1-2 \gamma}|\nabla \eta|_{g}^{2} v_{\alpha}^{2} d v_{g}+2 \int_{\partial^{\prime} \mathfrak{B}_{2 r}^{+}} \eta^{2}\left|v_{\alpha}\right|^{2^{*}} d \sigma_{\hat{h}}+o(1) \int_{\mathfrak{B}_{2 r}^{+}} \eta^{2}\left|v_{\alpha}\right| d v_{g} \\
& \quad \leq \frac{C}{r^{2}} \int_{\mathfrak{B}_{2 r}^{+}} \rho^{1-2 \gamma} v_{\alpha}^{2} d v_{g}+2 \int_{\partial^{\prime} \mathfrak{B}_{2 r}^{+}}\left(\eta v_{\alpha}\right)^{2}\left|v_{\alpha}\right|^{2^{*}-2} d \sigma_{\hat{h}}+o(1) \int_{\mathfrak{B}_{2 r}^{+}} \eta^{2}\left|v_{\alpha}\right| d v_{g} .
\end{aligned}
$$


By Hölder's inequality and our initial hypothesis,

$$
\begin{aligned}
\int_{\partial^{\prime} \mathfrak{B}_{2 r}^{+}}\left(\eta v_{\alpha}\right)^{2}\left|v_{\alpha}\right|^{2^{*}-2} d \sigma_{\hat{h}} & \leq\left(\int_{\partial^{\prime} \mathfrak{B}_{2 r}^{+}}\left|\eta v_{\alpha}\right|^{2^{*}} d \sigma_{\hat{h}}\right)^{\frac{2}{2^{*}}}\left(\int_{\partial^{\prime} \mathfrak{B}_{2 r}^{+}}\left|v_{\alpha}\right|^{2^{*}} d \sigma_{\hat{h}}\right)^{\frac{2^{*}-2}{2^{*}}} \\
& \leq \varepsilon^{\varepsilon^{2^{*}-2}}\left(\int_{\partial^{\prime} \mathfrak{B}_{2 r}^{+}}\left|\eta v_{\alpha}\right|^{2^{*}} d \sigma_{\hat{h}}\right)^{\frac{2}{2^{*}}} .
\end{aligned}
$$

Then it follows from above that

$$
\begin{aligned}
& \int_{\mathfrak{B}_{2 r}^{+}} \rho^{1-2 \gamma}\left|\nabla\left(\eta v_{\alpha}\right)\right|_{g}^{2} d v_{g} \\
& \quad \leq 2 \int_{\mathfrak{B}_{2 r}^{+}} \rho^{1-2 \gamma}\left(|\nabla \eta|_{g}^{2} v_{\alpha}^{2}+\eta^{2}\left|\nabla v_{\alpha}\right|_{g}^{2}\right) d v_{g} \\
& \quad \leq \frac{C}{r^{2}} \int_{\mathfrak{B}_{2 r}^{+}} \rho^{1-2 \gamma} v_{\alpha}^{2} d v_{g}+C \varepsilon^{\frac{2^{*}-2}{2^{*}}}\left(\int_{\partial^{\prime} \mathfrak{B}_{2 r}^{+}}\left|\eta v_{\alpha}\right|^{2^{*}} d \sigma_{\hat{h}}\right)^{\frac{2}{2^{*}}}+o(1) \int_{\mathfrak{B}_{2 r}^{+}} \eta^{2} v_{\alpha} d v_{g} .
\end{aligned}
$$

The trace Sobolev inequality on our manifold setting (Proposition 2.4) gives that

$$
\left(\int_{\partial^{\prime} \mathfrak{B}_{2 r}^{+}}\left|\eta v_{\alpha}\right|^{2^{*}} d \sigma_{\hat{h}}\right)^{\frac{2}{2^{*}}} \leq C \int_{\mathfrak{B}_{2 r}^{+}} \rho^{1-2 \gamma}\left|\nabla\left(\eta v_{\alpha}\right)\right|_{g}^{2} d v_{g}+C \int_{\partial^{\prime} \mathfrak{B}_{2 r}^{+}}\left(\eta v_{\alpha}\right)^{2} d \sigma_{\hat{h}} .
$$

Therefore,

$$
\begin{aligned}
& \int_{\mathfrak{B}_{2 r}^{+}} \rho^{1-2 \gamma}\left|\nabla\left(\eta v_{\alpha}\right)\right|_{g}^{2} d v_{g} \\
& \leq \frac{C}{r^{2}} \int_{\mathfrak{B}_{2 r}^{+}} \rho^{1-2 \gamma} v_{\alpha}^{2} d v_{g}+C \varepsilon^{\frac{2^{*}-2}{2^{*}}} \int_{\mathfrak{B}_{2 r}^{+}} \rho^{1-2 \gamma}\left|\nabla\left(\eta v_{\alpha}\right)\right|_{g}^{2} d v_{g} \\
& \quad+C \varepsilon^{\frac{2^{*}-2}{2^{*}}} \int_{\partial^{\prime} \mathfrak{B}_{2 r}^{+}}\left(\eta v_{\alpha}\right)^{2} d \sigma_{\hat{h}}+o(1) \int_{\mathfrak{B}_{2 r}^{+}} \eta^{2}\left|v_{\alpha}\right| d v_{g} .
\end{aligned}
$$

Now, fix $r>0$ small such that $\varepsilon$ is small enough to satisfy $C \varepsilon^{\frac{2^{*}-2}{2^{*}}} \leq \frac{1}{2}$. Then,

$$
\begin{aligned}
\int_{\mathfrak{B}_{r}^{+}} \rho^{1-2 \gamma} \mid \nabla & \left.v_{\alpha}\right|_{g} ^{2} d v_{g} \\
\leq & \frac{C}{r^{2}} \int_{\mathfrak{B}_{2 r}^{+}} \rho^{1-2 \gamma} v_{\alpha}^{2} d v_{g}+C \int_{\partial^{\prime} \mathfrak{B}_{2 r}^{+}} v_{\alpha}^{2} d \sigma_{\hat{h}}+o(1) \int_{\mathfrak{B}_{2 r}^{+}}\left|v_{\alpha}\right| d v_{g} .
\end{aligned}
$$

\section{The first bubble argument}

In this section, we focus on the blow up analysis of a Palais-Smale sequence which are not strongly convergent. In particular, using the $\varepsilon$-regularity estimates 
(Lemma 2.14), we can figure out the first bubble. We will also show that the PalaisSmale sequence obtained by subtracting a bubble is also Palais-Smale sequence and that the energy is splitting.

Lemma 3.1. Let $\left\{\hat{u}_{\alpha}\right\}_{\alpha \in \mathbb{N}}$ be a Palais-Smale sequence for $I_{g}^{\gamma}$ such that $\hat{u}_{\alpha} \rightarrow 0$ weakly in $W^{1,2}\left(X, \rho^{1-2 \gamma}\right)$, but not strongly as $\alpha \rightarrow+\infty$. Then, there exists a sequence of real numbers $\left\{\mu_{\alpha}>0\right\}_{\alpha \in \mathbb{N}}, \mu_{\alpha} \rightarrow 0$ as $\alpha \rightarrow+\infty$, a converging sequence of points $\left\{x_{\alpha}\right\}_{\alpha \in \mathbb{N}} \subset M$, and a nontrivial solution $u$ to the equation

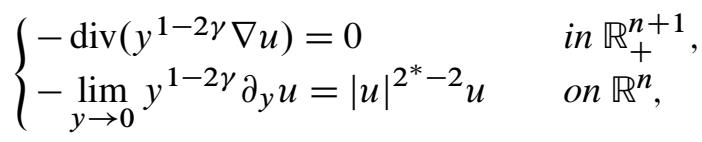

such that, up to a subsequence, if we take

$$
\hat{v}_{\alpha}(z)=\hat{u}_{\alpha}(z)-\eta_{\alpha}(z) \mu_{\alpha}^{-\frac{n-2 \gamma}{2}} u\left(\mu_{\alpha}^{-1} \varphi_{x_{\alpha}}^{-1}(z)\right), \quad z \in \varphi_{x_{\alpha}}\left(B_{2 r_{0}}^{+}(0)\right),
$$

where $r_{0}, \eta_{\alpha}(z)$, and $\varphi_{x_{\alpha}}(z)$ are the same as in Theorem 1.3, then we have the following three conclusions:

(1) $\hat{v}_{\alpha} \rightarrow 0$ weakly in $W^{1,2}\left(X, \rho^{1-2 \gamma}\right)$ as $\alpha \rightarrow+\infty$;

(2) $\left\{\hat{v}_{\alpha}\right\}_{\alpha \in \mathbb{N}}$ is also a Palais-Smale sequence for $I_{g}^{\gamma}$;

(3) $I_{g}^{\gamma}\left(\hat{v}_{\alpha}\right)=I_{g}^{\gamma}\left(\hat{u}_{\alpha}\right)-\widetilde{E}(u)+o(1)$ as $\alpha \rightarrow+\infty$.

Proof. Without loss of generality, we assume that $\hat{u}_{\alpha} \in \mathscr{C}^{\infty}(\bar{X})$. By the proof of Lemma 2.13,

$$
I_{g}^{\gamma}\left(\hat{u}_{\alpha}\right)=\frac{\gamma}{n} \int_{X} \rho^{1-2 \gamma}\left|\nabla \hat{u}_{\alpha}\right|_{g}^{2} d v_{g}+o(1)=\frac{\gamma}{n} \int_{M}\left|\hat{u}_{\alpha}\right|^{2^{*}} d \sigma_{\hat{h}}+o(1) .
$$

Note that $\left\{\hat{u}_{\alpha}\right\}_{\alpha \in \mathbb{N}}$ is uniformly bounded in $W^{1,2}\left(X, \rho^{1-2 \gamma}\right)$ by Lemma 2.9 , so there exist a subsequence, also denoted by $\left\{\hat{u}_{\alpha}\right\}_{\alpha \in \mathbb{N}}$ and a nonnegative constant $\beta$, such that

$$
I_{g}^{\gamma}\left(\hat{u}_{\alpha}\right)=\beta+o(1), \quad \text { as } \alpha \rightarrow+\infty .
$$

Since $\hat{u}_{\alpha} \rightarrow 0$ weakly in $W^{1,2}\left(X, \rho^{1-2 \gamma}\right)$ but not strongly as $\alpha \rightarrow+\infty$, again by Lemma 2.13,

$$
\lim _{\alpha \rightarrow+\infty} \int_{M}\left|\hat{u}_{\alpha}\right|^{2^{*}} d \sigma_{\hat{h}}=\frac{n}{\gamma} \beta \geq \frac{n}{\gamma} \beta_{0} .
$$

We will decompose the rest of the proof into several steps:

Step 1. Pick up the likely blow up points.

Claim 1. For any $t_{0}>0$ small, there exist $x_{0} \in M$ and $\varepsilon_{0}>0$ such that, up to a subsequence,

$$
\int_{\mathfrak{D}_{t_{0}}\left(x_{0}\right)}\left|\hat{u}_{\alpha}\right|^{2^{*}} d \sigma_{\hat{h}} \geq \varepsilon_{0}
$$


Proof. If the claim is not true, then there exists $t>0$ small, such that for any $x \in M$,

$$
\int_{\mathfrak{D}_{t}(x)}\left|\hat{u}_{\alpha}\right|^{2^{*}} d \sigma_{\hat{h}} \rightarrow 0, \quad \alpha \rightarrow+\infty .
$$

On the other hand, since $(M, \hat{h})$ is compact and $M \subset \bigcup_{x \in M} \mathfrak{D}_{t}(x)$, there exists an integer $N \geq 1$ such that $M \subset \bigcup_{i=1}^{N} \mathfrak{D}_{t}\left(x_{i}\right)$. Thus,

$$
\int_{M}\left|\hat{u}_{\alpha}\right|^{2^{*}} d \sigma_{\hat{h}} \leq \sum_{i=1}^{N} \int_{\mathfrak{D}_{t}\left(x_{i}\right)}\left|\hat{u}_{\alpha}\right|^{2^{*}} d \sigma_{\hat{h}} \rightarrow 0, \quad \alpha \rightarrow+\infty,
$$

which is a contradiction.

For $t>0$, we set

$$
\omega_{\alpha}(t)=\max _{x \in M} \int_{\mathfrak{D}_{t}(x)}\left|\hat{u}_{\alpha}\right|^{2^{*}} d \sigma_{\hat{h}}
$$

Then, by Claim 1, there exists $x_{\alpha} \in M$ such that

$$
\omega_{\alpha}\left(t_{0}\right)=\int_{\mathfrak{D}_{t_{0}}\left(x_{\alpha}\right)}\left|\hat{u}_{\alpha}\right|^{2^{*}} d \sigma_{\hat{h}} \geq \varepsilon_{0} .
$$

Note that

$$
\int_{\mathfrak{D}_{t}\left(x_{\alpha}\right)}\left|\hat{u}_{\alpha}\right|^{2^{*}} d \sigma_{\hat{h}} \rightarrow 0, \quad \text { as } t \rightarrow 0 .
$$

Hence, for any $\varepsilon \in\left(0, \varepsilon_{0}\right)$, there exists $t_{\alpha} \in\left(0, t_{0}\right)$ such that

$$
\varepsilon=\int_{\mathfrak{D}_{t_{\alpha}}\left(x_{\alpha}\right)}\left|\hat{u}_{\alpha}\right|^{2^{*}} d \sigma_{\hat{h}}
$$

Step 2. At each likely blow up point, we will establish weak convergence of a Palais-Smale sequence after properly rescaling.

For $r_{0}>0$ small, consider the Fermi coordinates at the likely blow up point $x_{\alpha} \in M, \varphi_{x_{\alpha}}: B_{2 r_{0}}^{+}(0) \rightarrow X$. Here we restrict $r_{0}$ to $r_{0} \leq i_{g}(X) / 2$, where $i_{g}(X)$ is the injectivity radius of $X$. Then, for any $0<\mu_{\alpha} \leq 1$, we define

$$
\begin{aligned}
& \tilde{u}_{\alpha}(z)=\mu_{\alpha}^{(n-2 \gamma) / 2} \hat{u}_{\alpha}\left(\varphi_{x_{\alpha}}\left(\mu_{\alpha} z\right)\right), \\
& \tilde{g}_{\alpha}(z)=\left(\varphi_{x_{\alpha}}^{*} g\right)\left(\mu_{\alpha} z\right), \\
& \tilde{h}_{\alpha}(x)=\left(\varphi_{x_{\alpha}}^{*} \hat{h}\right)\left(\mu_{\alpha} x\right),
\end{aligned}
$$

if $z \in B_{\mu_{\alpha}^{-1} r_{0}}^{+}(0)$ and $x \in \partial^{\prime} B_{\mu_{\alpha}^{-1} r_{0}}^{+}(0)$.

Given $z_{0} \in \mathbb{R}_{+}^{n+1}$ and $r>0$ such that $\left|z_{0}\right|+r<\mu_{\alpha}^{-1} r_{0}$, we have

$$
\int_{B_{r}^{+}\left(z_{0}\right)} \tilde{\rho}_{\alpha}^{1-2 \gamma}\left|\nabla \tilde{u}_{\alpha}\right|_{\tilde{g}_{\alpha}}^{2} d v_{\tilde{g}_{\alpha}}=\int_{\varphi_{x_{\alpha}}\left(\mu_{\alpha} B_{r}^{+}\left(z_{0}\right)\right)} \rho^{1-2 \gamma}\left|\nabla \hat{u}_{\alpha}\right|_{g}^{2} d v_{g},
$$


where

$$
\tilde{\rho}_{\alpha}(z)=\mu_{\alpha}^{-1} \rho\left(\varphi_{x_{\alpha}}\left(\mu_{\alpha} z\right)\right)
$$

and $\left|d \tilde{\rho}_{\alpha}\right|_{\tilde{g}_{\alpha}}=1$ on $\partial^{\prime} B_{r}^{+}\left(z_{0}\right)$ since $|d \rho|_{g}=1$ on $M$.

On the other hand, if $z_{0} \in \mathbb{R}^{n}$, and $\left|z_{0}\right|+r<\mu_{\alpha}^{-1} r_{0}$, then

$$
\int_{D_{r}\left(z_{0}\right)}\left|\tilde{u}_{\alpha}\right|^{2^{*}} d \sigma_{\tilde{h}_{\alpha}}=\int_{\varphi_{x_{\alpha}}\left(\mu_{\alpha} D_{r}\left(z_{0}\right)\right)}\left|\hat{u}_{\alpha}\right|^{2^{*}} d \sigma_{\hat{h}} \leq \int_{\mathfrak{D}_{2 \mu_{\alpha} r}\left(\varphi_{x_{\alpha}}\left(\mu_{\alpha} z_{0}\right)\right)}\left|\hat{u}_{\alpha}\right|^{2^{*}} d \sigma_{\hat{h}} .
$$

Here we have used that $\varphi_{x_{\alpha}}\left(\mu_{\alpha} D_{r}\left(z_{0}\right)\right)=\varphi_{x_{\alpha}}\left(D_{\mu_{\alpha} r}\left(\mu_{\alpha} z_{0}\right)\right)$, and for $x, y \in \mathbb{R}^{n}$, with $|x|<r_{0},|y|<r_{0}$, we have $\frac{1}{2}|x-y| \leq d_{g}\left(\varphi_{x_{\alpha}}(x), \varphi_{x_{\alpha}}(y)\right) \leq 2|x-y|$.

Next, take $r \in\left(0, r_{0}\right)$ and choose $t_{0}$ in Claim 1 such that $0<t_{0} \leq 2 r$. For any $\varepsilon \in\left(0, \varepsilon_{0}\right)$, with $\varepsilon$ to be determined later, and $t_{\alpha} \in\left(0, t_{0}\right)$, let

$$
0<\mu_{\alpha}=\frac{1}{2} r^{-1} t_{\alpha} \leq \frac{1}{2} r^{-1} t_{0} \leq 1 .
$$

Then, by the definition of $\varepsilon$ from (3-2), if $\left|z_{0}\right|+r<\mu_{\alpha}^{-1} r_{0}$,

$$
\int_{\partial^{\prime} B_{r}^{+}\left(z_{0}\right)}\left|\tilde{u}_{\alpha}\right|^{2^{*}} d \sigma_{\tilde{h}_{\alpha}} \leq \varepsilon
$$

Note that $\varphi_{x_{\alpha}}\left(\partial^{\prime} B_{2 r \mu_{\alpha}}^{+}(0)\right)=\mathfrak{D}_{t_{\alpha}}\left(x_{\alpha}\right)$, we have

$$
\begin{aligned}
\varepsilon=\int_{\mathfrak{D}_{t \alpha}\left(x_{\alpha}\right)}\left|\hat{u}_{\alpha}\right|^{2^{*}} d \sigma_{\hat{h}}=\int_{\varphi_{x_{\alpha}}\left(\partial^{\prime} B_{2 r \mu \alpha}^{+}(0)\right)}\left|\hat{u}_{\alpha}\right|^{2^{*}} d \sigma_{\hat{h}} \\
\quad=\int_{\varphi_{x_{\alpha}}\left(\mu_{\alpha} \partial^{\prime} B_{2 r}^{+}(0)\right)}\left|\hat{u}_{\alpha}\right|^{2^{*}} d \sigma_{\hat{h}}=\int_{\partial^{\prime} B_{2 r}^{+}(0)}\left|\tilde{u}_{\alpha}\right|^{2^{*}} d \sigma_{\tilde{h}_{\alpha}} .
\end{aligned}
$$

This $r_{0}>0$ can be chosen smaller again, such that for any $0<\mu \leq 1$ and any $x_{0} \in M$, we can assume that

$$
\begin{aligned}
\frac{1}{2} \int_{\mathbb{R}_{+}^{n+1}} y^{1-2 \gamma}|\nabla u|^{2} d x d y & \leq \int_{\mathbb{R}_{+}^{n+1}} \tilde{\rho}_{x_{0}, \mu}^{1-2 \gamma}|\nabla u|_{\tilde{g}_{x_{0}, \mu}}^{2} d v_{\tilde{g}_{x_{0}, \mu}} \\
& \leq 2 \int_{\mathbb{R}_{+}^{n+1}} y^{1-2 \gamma}|\nabla u|^{2} d x d y,
\end{aligned}
$$

where $u \in \bar{W}^{1,2}\left(\mathbb{R}_{+}^{n+1}, \tilde{\rho}_{x_{0}, \mu}^{1-2 \gamma}\right), \operatorname{supp}(u) \subset B_{2 \mu^{-1} r_{0}}^{+}(0), \tilde{\rho}_{x_{0}, \mu}(z)=\mu^{-1} \rho\left(\varphi_{x_{0}}(\mu z)\right)$, and $\tilde{g}_{x_{0}, \mu}(z)=\left(\varphi_{x_{0}}^{*} g\right)(\mu z)$. And for $u \in L^{1}\left(\mathbb{R}^{n}\right)$ such that $\operatorname{supp}(u) \subset \partial^{\prime} B_{2 \mu}^{+}{ }^{-1} r_{0}(0)$, we can also assume that

$$
\frac{1}{2} \int_{\mathbb{R}^{n}}|u| d x \leq \int_{\mathbb{R}^{n}}|u| d \sigma_{\tilde{h}_{x_{0}, \mu}} \leq 2 \int_{\mathbb{R}^{n}}|u| d x,
$$

where $\tilde{h}_{x_{0}, \mu}(x)=\left(\varphi_{x_{0}}^{*} \hat{h}\right)(\mu x)$. 
Let $\tilde{\eta} \in \mathscr{C}_{0}^{\infty}\left(\mathbb{R}_{+}^{n+1}\right)$ be a cutoff function satisfying $0 \leq \tilde{\eta} \leq 1$,

$$
\tilde{\eta} \equiv \begin{cases}1, & \text { in } B_{1 / 4}^{+}(0), \\ 0, & \text { in } \mathbb{R}_{+}^{n+1} \backslash B_{3 / 4}^{+}(0),\end{cases}
$$

and set $\tilde{\eta}_{\alpha}(z)=\tilde{\eta}\left(r_{0}^{-1} \mu_{\alpha} z\right)$.

Claim 2. $\left\{\tilde{\eta}_{\alpha} \tilde{u}_{\alpha}\right\}_{\alpha \in \mathbb{N}}$ is uniformly bounded in $W^{1,2}\left(\mathbb{R}_{+}^{n+1}, y^{1-2 \gamma}\right)$.

Proof. Note that

$$
\begin{aligned}
\int_{\mathbb{R}_{+}^{n+1}} & \tilde{\rho}_{\alpha}^{1-2 \gamma}\left|\nabla\left(\tilde{\eta}_{\alpha} \tilde{u}_{\alpha}\right)\right|_{\tilde{g}_{\alpha}}^{2} d v_{\tilde{g}_{\alpha}}+\int_{\mathbb{R}_{+}^{n+1}} \tilde{\rho}_{\alpha}^{1-2 \gamma}\left(\tilde{\eta}_{\alpha} \tilde{u}_{\alpha}\right)^{2} d v_{\tilde{g}_{\alpha}} \\
& \leq \int_{\mathbb{R}_{+}^{n+1}} \tilde{\rho}_{\alpha}^{1-2 \gamma}\left(2\left|\nabla \tilde{\eta}_{\alpha}\right|_{\tilde{g}_{\alpha}}^{2}+\tilde{\eta}_{\alpha}^{2}\right) \tilde{u}_{\alpha}^{2} d v_{\tilde{g}_{\alpha}}+2 \int_{\mathbb{R}_{+}^{n+1}} \tilde{\rho}_{\alpha}^{1-2 \gamma} \tilde{\eta}_{\alpha}^{2}\left|\nabla \tilde{u}_{\alpha}\right|_{\tilde{g}_{\alpha}}^{2} d v_{\tilde{g}_{\alpha}} \\
& \leq C \int_{X} \rho^{1-2 \gamma} \hat{u}_{\alpha}^{2} d v_{g}+C \int_{X} \rho^{1-2 \gamma}\left|\nabla \hat{u}_{\alpha}\right|_{g}^{2} d v_{g} \leq C,
\end{aligned}
$$

since $\left\{\hat{u}_{\alpha}\right\}_{\alpha \in \mathbb{N}}$ is uniformly bounded in $W^{1,2}\left(X, \rho^{1-2 \gamma}\right)$. Combining this with (3-4), we get that $\left\{\tilde{\eta}_{\alpha} \tilde{u}_{\alpha}\right\}_{\alpha \in \mathbb{N}}$ is uniformly bounded in $W^{1,2}\left(\mathbb{R}_{+}^{n+1}, y^{1-2 \gamma}\right)$.

Due to the weak compactness of $W^{1,2}\left(\mathbb{R}_{+}^{n+1}, y^{1-2 \gamma}\right)$, there exists some $u$ in $W^{1,2}\left(\mathbb{R}_{+}^{n+1}, y^{1-2 \gamma}\right)$ such that $\tilde{\eta}_{\alpha} \tilde{u}_{\alpha} \rightarrow u$ in $W^{1,2}\left(\mathbb{R}_{+}^{n+1}, y^{1-2 \gamma}\right)$ as $\alpha \rightarrow+\infty$.

Step 3. The weak convergence is in fact strong via $\varepsilon$-regularity estimates.

Claim 3. Let $r_{1}=\frac{r_{0}}{8}$. Then, there exists $\varepsilon_{1}=\varepsilon_{1}(\gamma, n)$ such that for any $0<r<r_{1}$, $0<\varepsilon<\min \left\{\varepsilon_{0}, \varepsilon_{1}\right\}$, we have $\tilde{\eta}_{\alpha} \tilde{u}_{\alpha} \rightarrow u$ in $W^{1,2}\left(B_{2 r}^{+}(0), y^{1-2 \gamma}\right)$ as $\alpha \rightarrow+\infty$.

Proof. Given $r$ sufficiently small, to be determined later, for any $z_{0} \in \mathbb{R}_{+}^{n+1}$, let $\psi \in \mathscr{C}_{0}^{\infty}\left(B_{r}^{+}\left(z_{0}\right)\right) \cap W^{1,2}\left(\mathbb{R}_{+}^{n+1}, y^{1-2 \gamma}\right)$. Let

$$
\hat{\psi}_{\alpha}(z)=\mu_{\alpha}^{-\frac{n-2 \gamma}{2}} \psi\left(\mu_{\alpha}^{-1} \varphi_{x_{\alpha}}^{-1}(z)\right) \quad \text { for } z \in \varphi_{x_{\alpha}}\left(B_{r}^{+}\left(z_{0}\right)\right) .
$$

Since $\left\{\hat{u}_{\alpha}\right\}$ satisfies the asymptotic equation (2-10),

$$
\begin{aligned}
o(1)\|\psi\|_{\bar{W}^{1,2}\left(\mathbb{R}_{+}^{n+1}, y^{1-2 \gamma)}\right)} & o(1)\left\|\hat{\psi}_{\alpha}\right\|_{\bar{W}^{1,2}\left(X, \rho^{1-2 \gamma}\right)} \\
& =\int_{\varphi_{x_{\alpha}}\left(\mu_{\alpha} B_{r}^{+}\left(z_{0}\right)\right)} \rho^{1-2 \gamma}\left\langle\nabla \hat{u}_{\alpha}, \nabla \hat{\psi}_{\alpha}\right\rangle_{g} d v_{g} \\
& =\int_{B_{r}^{+}\left(z_{0}\right)}\left(\mu_{\alpha}^{-1} \rho\right)^{1-2 \gamma}\left\langle\nabla\left(\tilde{\eta}_{\alpha} \tilde{u}_{\alpha}\right), \nabla \psi\right\rangle_{\tilde{g}_{\alpha}} d v_{\tilde{g}_{\alpha}},
\end{aligned}
$$

since $\tilde{\eta}$ is supported in $B_{3 / 4}^{+}(0)$ and $\tilde{\eta} \equiv 1$ in $B_{1 / 4}^{+}(0)$. Also, note that since $\tilde{\eta}_{\alpha}(z)=$ $\tilde{\eta}\left(\mu_{\alpha} r_{0}^{-1} z\right)$, we have $\tilde{\eta}_{\alpha} \equiv 1$ in $B_{1 / 4 \mu_{\alpha}^{-1}}^{+} r_{0}$; thus, we need $\left|z_{0}\right|+r<\frac{1}{4} \mu_{\alpha}^{-1} r_{0}$. 
It is easy to check that $\mu_{\alpha}^{-1} \rho \rightarrow y$ as $\alpha \rightarrow+\infty$ since $\left|d\left(\mu_{\alpha}^{-1} \rho\right)\right|_{\tilde{g}_{\alpha}}=1$ on $\mathbb{R}^{n}$ and $\tilde{g}_{\alpha} \rightarrow\left(|d x|^{2}+d y^{2}\right)$. Then we have the asymptotic equation

$$
-\operatorname{div}\left(y^{1-2 \gamma} \nabla\left(\tilde{\eta}_{\alpha} \tilde{u}_{\alpha}\right)\right)=o(1) \text { in } B_{r}^{+}\left(z_{0}\right) .
$$

Since $\tilde{\eta}_{\alpha} \tilde{u}_{\alpha} \rightarrow u$ weakly in $W^{1,2}\left(\mathbb{R}_{+}^{n+1}, y^{1-2 \gamma}\right)$, we simultaneously get that

$$
-\operatorname{div}\left(y^{1-2 \gamma} \nabla u\right)=0 \quad \text { in } B_{r}^{+}\left(z_{0}\right) .
$$

Now, let $\psi \in W^{1,2}\left(B_{r}^{+}\left(z_{0}\right), y^{1-2 \gamma}\right)$. Then, multiplying both sides of (3-5) by $\psi$ and integrating by parts, we get

$$
\begin{aligned}
o(1)\|\psi\|_{W^{1,2}\left(B_{r}^{+}\left(z_{0}\right), y^{1-2 \gamma}\right)}= & \int_{\partial^{\prime} B_{r}^{+}\left(z_{0}\right)} \lim _{y \rightarrow 0} y^{1-2 \gamma} \partial_{y}\left(\tilde{\eta}_{\alpha} \tilde{u}_{\alpha}\right) \psi d \sigma_{\tilde{h}_{\alpha}} \\
& +\int_{B_{r}^{+}\left(z_{0}\right)} y^{1-2 \gamma}\left\langle\nabla\left(\tilde{\eta}_{\alpha} \tilde{u}_{\alpha}\right), \nabla \psi\right\rangle_{\tilde{g}_{\alpha}} d v_{\tilde{g}_{\alpha}} .
\end{aligned}
$$

On the other hand, using (2-10), (2-11), and the definition of $\hat{\psi}_{\alpha}$,

$$
\begin{aligned}
\int_{B_{r}^{+}\left(z_{0}\right)} & y^{1-2 \gamma}\left\langle\nabla\left(\tilde{\eta}_{\alpha} \tilde{u}_{\alpha}\right), \nabla \psi\right\rangle_{\tilde{g}_{\alpha}} d v_{\tilde{g}_{\alpha}} \\
= & \int_{\varphi_{X_{\alpha}}\left(\mu_{\alpha} B_{r}^{+}\left(z_{0}\right)\right)} \rho^{1-2 \gamma}\left\langle\nabla \hat{u}_{\alpha}, \nabla \hat{\psi}_{\alpha}\right\rangle_{g} d v_{g} \\
= & -\int_{M} \lim _{\rho \rightarrow 0} \rho^{1-2 \gamma}\left(\partial_{\rho} \hat{u}_{\alpha}\right) \psi_{\alpha} d \sigma_{\hat{h}}+o(1)\left\|\hat{\psi}_{\alpha}\right\|_{W^{1,2}\left(X, \rho^{1-2 \gamma}\right)} \\
= & \int_{M}\left|\hat{u}_{\alpha}\right|^{2^{*}-2} \hat{u}_{\alpha} \hat{\psi}_{\alpha} d \sigma_{\hat{h}}+o(1)\left\|\hat{\psi}_{\alpha}\right\|_{W^{1,2}\left(X, \rho^{1-2 \gamma}\right)} \\
= & \int_{\partial^{\prime} B_{r}^{+}\left(z_{0}\right)}\left|\tilde{\eta}_{\alpha} \tilde{u}_{\alpha}\right|^{2^{*}-2}\left(\tilde{\eta}_{\alpha} \tilde{u}_{\alpha}\right) \psi d \sigma_{\tilde{h}_{\alpha}}+o(1)\left\|\hat{\psi}_{\alpha}\right\|_{W^{1,2}\left(X, \rho^{1-2 \gamma}\right)} .
\end{aligned}
$$

Since $\|\psi\|_{W^{1,2}\left(B_{r}^{+}\left(z_{0}\right), y^{1-2 \gamma}\right)}=\left\|\hat{\psi}_{\alpha}\right\|_{W^{1,2}\left(X, \rho^{1-2 \gamma}\right)}$, combining expressions (3-7) and (3-8) yields

$$
\begin{aligned}
o(1)\|\psi\|_{W^{1,2}\left(B_{r}^{+}\left(z_{0}\right), y^{1-2 \gamma)}\right.}= & \int_{\partial^{\prime} B_{r}^{+}\left(z_{0}\right)} \lim _{y \rightarrow 0} y^{1-2 \gamma} \partial_{y}\left(\tilde{\eta}_{\alpha} \tilde{u}_{\alpha}\right) \psi d \sigma_{\tilde{h}_{\alpha}} \\
& +\int_{\partial^{\prime} B_{r}^{+}\left(z_{0}\right)}\left|\tilde{\eta}_{\alpha} \tilde{u}_{\alpha}\right|^{2^{*}-2}\left(\tilde{\eta}_{\alpha} \tilde{u}_{\alpha}\right) \psi d \sigma_{\tilde{h}_{\alpha}},
\end{aligned}
$$

i.e.,

$$
-\lim _{y \rightarrow 0} y^{1-2 \gamma} \partial_{y}\left(\tilde{\eta}_{\alpha} \tilde{u}_{\alpha}\right)=\left|\tilde{\eta}_{\alpha} \tilde{u}_{\alpha}\right|^{2^{*}-2}\left(\tilde{\eta}_{\alpha} \tilde{u}_{\alpha}\right)+o(1) \quad \text { on } \partial^{\prime} B_{r}^{+}\left(z_{0}\right) \text {. }
$$


Meanwhile, since $\tilde{\eta}_{\alpha} \tilde{u}_{\alpha} \rightarrow u$ weakly in $W^{1,2}\left(\mathbb{R}_{+}^{n+1}, y^{1-2 \gamma}\right)$, the same argument as above gives that

$$
-\lim _{y \rightarrow 0} y^{1-2 \gamma} \partial_{y} u=|u|^{2^{*}-2} u \quad \text { on } \partial^{\prime} B_{r}^{+}\left(z_{0}\right) .
$$

If we denote by

$$
\Gamma_{\alpha}:=\left|\tilde{\eta}_{\alpha} \tilde{u}_{\alpha}\right|^{2^{*}-2}\left(\tilde{\eta}_{\alpha} \tilde{u}_{\alpha}\right)-|u|^{2^{*}-2} u-\left|\tilde{\eta}_{\alpha} \tilde{u}_{\alpha}-u\right|^{2^{*}-2}\left(\tilde{\eta}_{\alpha} \tilde{u}_{\alpha}-u\right),
$$

then

$$
\begin{cases}-\operatorname{div}\left(y^{1-2 \gamma} \nabla\left(\tilde{\eta}_{\alpha} \tilde{u}_{\alpha}-u\right)\right)=o(1) & \text { in } B_{r}^{+}\left(z_{0}\right), \\ -\lim _{y \rightarrow 0} y^{1-2 \gamma} \partial_{y}\left(\tilde{\eta}_{\alpha} \tilde{u}_{\alpha}-u\right) & \\ =\left|\tilde{\eta}_{\alpha} \tilde{u}_{\alpha}-u\right|^{2^{*}-2}\left(\tilde{\eta}_{\alpha} \tilde{u}_{\alpha}-u\right)+\Gamma_{\alpha}+o(1) & \text { on } \partial^{\prime} B_{r}^{+}\left(z_{0}\right) .\end{cases}
$$

We have proved in (3-3) that for any $r>0$ and $\varepsilon_{1}>0$, there exists a sequence $\left\{\mu_{\alpha}\right\}_{\alpha \in \mathbb{N}}$ such that, if $\left|z_{0}\right|+r \leq r_{0} \leq \mu_{\alpha}^{-1} r_{0}$, then

$$
\int_{\partial^{\prime} B_{r}^{+}\left(z_{0}\right)}\left|\tilde{u}_{\alpha}\right|^{2^{*}} d x \leq \varepsilon_{1} \text {. }
$$

Therefore, we can also choose $r$ small enough such that, if $\left|z_{0}\right|+3 r<r_{0}$, then

$$
\int_{\partial^{\prime} B_{r}^{+}\left(z_{0}\right)}\left|\tilde{\eta}_{\alpha} \tilde{u}_{\alpha}-u\right|^{2^{*}} d x \leq \varepsilon_{1} .
$$

We claim that $\Gamma_{\alpha}=o(1)$ in the sense that for any $\theta \in W^{1,2}\left(\mathbb{R}_{+}^{n+1}, y^{1-2 \gamma}\right)^{\prime}$,

$$
\int_{\partial^{\prime} B_{r}^{+}\left(z_{0}\right)}\left|\Gamma_{\alpha} \theta\right| d \sigma_{\hat{h}}=o(1)\|\theta\|_{L^{2^{*}}\left(\partial^{\prime} B_{r}^{+}\left(z_{0}\right)\right)}, \quad \text { as } \alpha \rightarrow+\infty .
$$

We can use the same arguments as in the proof of Lemma 2.12 to show this claim.

Then by the $\varepsilon$-regularity estimates and the compact embedding of the weighted Sobolev space, we can prove that $\tilde{\eta}_{\alpha} \tilde{u}_{\alpha} \rightarrow u$ in $W^{1,2}\left(B_{r}^{+}\left(z_{0}\right), y^{1-2 \gamma}\right)$. Then, by the finite covering we can prove that $\tilde{\eta}_{\alpha} \tilde{u}_{\alpha} \rightarrow u$ in $W^{1,2}\left(B_{2 r}^{+}(0), y^{1-2 \gamma}\right)$.

Applying Claim 3, noting that $\tilde{\eta}_{\alpha} \tilde{u}_{\alpha} \rightarrow u$ in $W^{1,2}\left(B_{2 r}^{+}(0), y^{1-2 \gamma}\right)$ and that $\tilde{\eta}_{\alpha} \equiv 1$ in $\partial^{\prime} B_{1 / 4 \mu_{\alpha}^{-1} r_{0}}^{+}$since $0<\mu_{\alpha} \leq 1$ and $2 r<\frac{r_{0}}{4}$,

$$
\varepsilon=\int_{\partial^{\prime} B_{2 r}^{+}(0)}\left|\tilde{u}_{\alpha}\right|^{2^{*}} d \sigma_{\tilde{h}_{\alpha}}=\int_{\partial^{\prime} B_{2 r}^{+}(0)}\left|\tilde{\eta}_{\alpha} \tilde{u}_{\alpha}\right|^{2^{*}} d \sigma_{\tilde{h}_{\alpha}} \leq 2 \int_{\partial^{\prime} B_{2 r}^{+}(0)}|u|^{2^{*}} d x+o(1),
$$

where we used $\tilde{\eta}_{\alpha} \tilde{u}_{\alpha} \rightarrow u$ in $L^{2^{*}}\left(\partial^{\prime} B_{2 r}^{+}(0),|d x|^{2}\right)$ as $\alpha \rightarrow+\infty$ by Proposition 2.4. So, $u \neq 0$.

Claim 4. $\lim _{\alpha \rightarrow+\infty} \mu_{\alpha}=0$.

In fact, if $\mu_{\alpha} \rightarrow \mu_{0}>0$, then $\tilde{\eta}_{\alpha} \tilde{u}_{\alpha} \rightarrow 0$ in $W^{1,2}\left(B_{2 r}^{+}(0), y^{1-2 \gamma}\right)$ since $\hat{u}_{\alpha} \rightarrow 0$ in $W^{1,2}\left(X, \rho^{1-2 \gamma}\right)$. But, $u \neq 0$, which is a contradiction. 
Claim 5. For any $0<\mu_{0} \leq 1, \tilde{u}_{\alpha} \rightarrow u$ strongly in $W^{1,2}\left(B_{\mu_{0}^{-1}}^{+}(0), y^{1-2 \gamma}\right)$ as $\alpha \rightarrow+\infty$, and $u$ is a weak solution of (3-1).

Proof. Let $0<\mu_{0} \leq 1$. By Claim 4, we know that $0<\mu_{\alpha} \leq \mu_{0}$ for $\alpha$ large. Then, (3-3) holds for $\left|z_{0}\right|+r<\mu_{0}^{-1} r_{0}$. By the same arguments, it is easy to check that

$$
\tilde{\eta}_{\alpha} \tilde{u}_{\alpha} \rightarrow u \quad \text { in } W^{1,2}\left(B_{2 r \mu_{0}^{-1}}^{+}(0), y^{1-2 \gamma}\right) .
$$

For $\alpha$ large, $\tilde{\eta}_{\alpha} \equiv 1$ in $B_{2 r}^{+} \mu_{0}^{-1}(0)$, so we have

$$
\tilde{u}_{\alpha} \rightarrow u \text { in } W^{1,2}\left(B_{2 r \mu_{0}^{-1}}^{+}(0), y^{1-2 \gamma}\right)
$$

strongly as $\alpha \rightarrow+\infty$.

Finally, we claim that $u$ solves the boundary problem

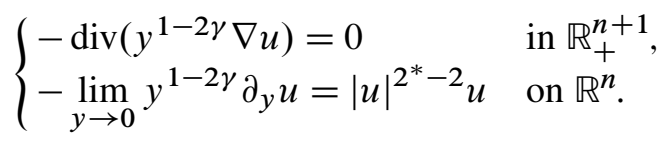

Since $0<\mu_{0} \leq 1$ is arbitrary, $\tilde{u}_{\alpha} \rightarrow u$ strongly in $W^{1,2}\left(B_{R}^{+}(0), y^{1-2 \gamma}\right)$ for any large $R>0$. Without loss of generality, let $\psi \in \mathscr{C}_{0}^{\infty}\left(\mathbb{R}_{+}^{n+1}\right)$ and $\operatorname{supp} \psi \subset B_{R_{0}}^{+}(0)$ for some $R_{0}>0$. Set

$$
\psi_{\alpha}(z)=\mu_{\alpha}^{-\frac{n-2 \gamma}{2}} \psi\left(\mu_{\alpha}^{-1} \varphi_{x_{\alpha}}^{-1}(z)\right) .
$$

For $\alpha$ large enough,

$$
\int_{X} \rho^{1-2 \gamma}\left\langle\nabla \hat{u}_{\alpha}, \nabla \psi_{\alpha}\right\rangle_{g} d v_{g}=\int_{\mathbb{R}_{+}^{n+1}} \tilde{\rho}_{\alpha}^{1-2 \gamma}\left\langle\nabla\left(\tilde{\eta}_{\alpha} \tilde{u}_{\alpha}\right), \nabla \psi\right\rangle_{\tilde{g}_{\alpha}} d v_{\tilde{g}_{\alpha}},
$$

and

$$
\int_{M}\left|\hat{u}_{\alpha}\right|^{2^{*}-2} \hat{u}_{\alpha} \psi_{\alpha} d v_{g}=\int_{\mathbb{R}^{n}}\left|\tilde{\eta}_{\alpha} \tilde{u}_{\alpha}\right|^{2^{*}-2}\left(\tilde{\eta}_{\alpha} \tilde{u}_{\alpha}\right) \psi d v_{\tilde{g}_{\alpha}} .
$$

Note that $\tilde{g}_{\alpha} \rightarrow|d x|^{2}+d y^{2}$ in $\mathscr{C}^{1}\left(B_{R}^{+}(0)\right)$ as $\alpha \rightarrow+\infty,\left\{\hat{u}_{\alpha}\right\}$ is a Palais-Smale sequence for $I_{g}^{\gamma}$ and $\tilde{\eta}_{\alpha} \tilde{u}_{\alpha} \rightarrow u$ in $W^{1,2}\left(B_{R}^{+}(0)\right)$ for any $R>0$. Then, we have

$$
\int_{\mathbb{R}_{+}^{n+1}} y^{1-2 \gamma}\langle\nabla u, \nabla \psi\rangle d x d y-\int_{\mathbb{R}^{n}}|u|^{2^{*}-2} u \psi d x d y=0,
$$

which yields our desired result.

Step 4. The Palais-Smale sequence minus a bubble is still a Palais-Smale sequence. $\overline{\text { Define }}$

$$
\begin{cases}\hat{w}_{\alpha}(z)=\hat{\eta}_{\alpha}(z) \mu_{\alpha}^{-\frac{n-2 \gamma}{2}} u\left(\mu_{\alpha}^{-1} \varphi_{x_{\alpha}}^{-1}(z)\right), & z \in \varphi_{x_{\alpha}}\left(B_{2 r_{0}}^{+}(0)\right), \\ \hat{w}_{\alpha}(z)=0, & \text { otherwise, }\end{cases}
$$

where $\hat{\eta}_{\alpha}$ is a cut-off function satisfying $\hat{\eta}_{\alpha}=1$ in $\varphi_{x_{\alpha}}\left(B_{r_{0}}^{+}(0)\right)$ and $\hat{\eta}_{\alpha}=0$ in $M \backslash \varphi_{x_{\alpha}}\left(B_{2 r_{0}}^{+}(0)\right)$. Here we have $\mathfrak{B}_{2 r_{0}}^{+}\left(x_{\alpha}\right)=\varphi_{x_{\alpha}}\left(B_{2 r_{0}}^{+}(0)\right)$. Let $\hat{v}_{\alpha}=\hat{u}_{\alpha}-\hat{w}_{\alpha}$. We claim: 
(i) $\hat{v}_{\alpha} \rightarrow 0$ in $W^{1,2}\left(X, \rho^{1-2 \gamma}\right)$ as $\alpha \rightarrow+\infty$;

(ii) $D I_{g}^{\gamma}\left(\hat{v}_{\alpha}\right) \rightarrow 0$ in $W^{1,2}\left(X, \rho^{1-2 \gamma}\right)^{\prime}$ as $\alpha \rightarrow+\infty$;

(iii) $I_{g}^{\gamma}\left(\hat{v}_{\alpha}\right)=I_{g}^{\gamma}\left(\hat{u}_{\alpha}\right)-\widetilde{E}(u)+o(1)$ as $\alpha \rightarrow+\infty$;

(iv) $\left\{\hat{v}_{\alpha}\right\}_{\alpha \in \mathbb{N}}$ is also a Palais-Smale sequence for $I_{g}^{\gamma}$.

The remainder of the proof of Lemma 3.1 consists of proving these claims.

(i) Since $\hat{u}_{\alpha} \rightarrow 0$ in $W^{1,2}\left(X, \rho^{1-2 \gamma}\right)$ as $\alpha \rightarrow+\infty$, it suffices to prove $\hat{w}_{\alpha} \rightarrow 0$ in $W^{1,2}\left(X, \rho^{1-2 \gamma}\right)$ as $\alpha \rightarrow+\infty$. First, we prove that $\int_{M} \hat{w}_{\alpha} \psi d \sigma_{\hat{h}}=o(1)$ as $\alpha \rightarrow+\infty$ for any $\psi \in \mathscr{C}^{\infty}(\bar{X})$. Given $R>0$,

$$
\int_{M} \hat{w}_{\alpha} \psi d \sigma_{\hat{h}}=\int_{\mathfrak{D}_{\mu_{\alpha} R}\left(x_{\alpha}\right)} \hat{w}_{\alpha} \psi d \sigma_{\hat{h}}+\int_{M \backslash \mathfrak{D}_{\mu_{\alpha} R}\left(x_{\alpha}\right)} \hat{w}_{\alpha} \psi d \sigma_{\hat{h}} .
$$

Note that $\tilde{h}_{\alpha}(x)=\left(\varphi_{x_{\alpha}}^{*} \hat{h}\right)\left(\mu_{\alpha} x\right)$. Using (3-11),

$$
\begin{aligned}
\int_{\mathfrak{D}_{\mu_{\alpha} R}\left(x_{\alpha}\right)} \hat{w}_{\alpha} \psi d \sigma_{\hat{h}} & =\int_{\mathfrak{D}_{\mu_{\alpha} R}\left(x_{\alpha}\right)} \hat{\eta}_{\alpha}(x) \mu_{\alpha}^{-\frac{n-2 \gamma}{2}} u\left(\mu_{\alpha}^{-1} \varphi_{x_{\alpha}}^{-1}(x)\right) \psi(x) d \sigma_{\hat{h}} \\
& =\mu_{\alpha}^{\frac{n+2 \gamma}{2}} \int_{D_{R}(0)} \hat{\eta}_{\alpha}\left(\varphi_{x_{\alpha}}\left(\mu_{\alpha} x\right)\right) u(x) \psi\left(\varphi_{x_{\alpha}}\left(\mu_{\alpha} x\right)\right) d \sigma_{\tilde{h}_{\alpha}} \\
& \leq C\|\psi\|_{L^{\infty}(M)} \mu_{\alpha}^{\frac{n+2 \gamma}{2}} \int_{D_{R}(0)}|u(x)| d x .
\end{aligned}
$$

Similarly, we can deal with the second term in the right hand side of (3-12):

$$
\begin{aligned}
& \int_{M \backslash \mathfrak{D}_{\mu_{\alpha} R}\left(x_{\alpha}\right)} \hat{w}_{\alpha} \psi d \sigma_{\hat{h}}=\int_{\mathfrak{D}_{2 r_{0}}\left(x_{\alpha}\right) \backslash \mathfrak{D}_{\mu_{\alpha} R}\left(x_{\alpha}\right)} \hat{w}_{\alpha} \psi d \sigma_{\hat{h}}
\end{aligned}
$$

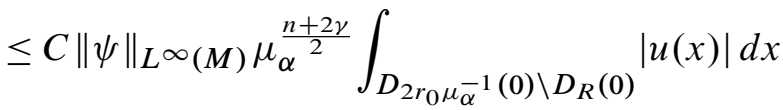

$$
\begin{aligned}
& \leq C\|\psi\|_{L^{\infty}(M)} \mu_{\alpha}^{\frac{n+2 \gamma}{2}}\left(\int_{D_{2 r_{0} \mu_{\alpha}^{-1}(0) \backslash D_{R}(0)}}|u(x)|^{2^{*}} d x\right)^{\frac{1}{2^{*}}}
\end{aligned}
$$

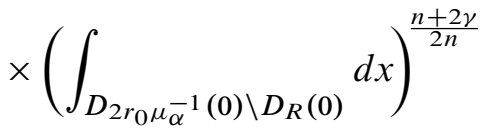

$$
\begin{aligned}
& \leq C\|\psi\|_{L^{\infty}(M)}\left(\int_{D_{2 r_{0} \mu_{\alpha}^{-1}(0) \backslash D_{R}(0)}}|u(x)|^{2^{*}} d x\right)^{\frac{1}{2^{*}}} .
\end{aligned}
$$

Since $u \in L^{2^{*}}\left(\mathbb{R}^{n},|d x|^{2}\right)$ and $\mu_{\alpha} \rightarrow 0$ as $\alpha \rightarrow+\infty$, taking $R$ large enough we get

$$
\int_{M} \hat{w}_{\alpha} \psi d \sigma_{\hat{h}}=o(1) \quad \text { as } \alpha \rightarrow+\infty \text {. }
$$


Next, we will show that

$$
\int_{X} \rho^{1-2 \gamma}\left\langle\nabla \hat{w}_{\alpha}, \nabla \psi\right\rangle_{g} d v_{g}=o(1) \quad \text { as } \alpha \rightarrow+\infty
$$

for any $\psi \in \mathscr{C}^{\infty}(\bar{X})$. Let $\tilde{\eta}_{\alpha}(z)=\hat{\eta}_{\alpha}\left(\varphi_{x_{\alpha}}\left(\mu_{\alpha} z\right)\right), \tilde{\rho}_{\alpha}(z)=\mu_{\alpha}^{-1} \rho\left(\varphi_{x_{\alpha}}\left(\mu_{\alpha} z\right)\right)$. Noting that $\hat{w}_{\alpha} \equiv 0$ in $X \backslash \mathfrak{B}_{2 r_{0}}^{+}\left(x_{\alpha}\right)$, then for any $R>0$ and $\alpha$ large,

$$
\begin{aligned}
\int_{X} \rho^{1-2 \gamma}\left\langle\nabla \hat{w}_{\alpha}, \nabla \psi\right\rangle_{g} d v_{g}= & \int_{\mathfrak{B}_{2 r_{0}}^{+}\left(x_{\alpha}\right)} \rho^{1-2 \gamma}\left\langle\nabla \hat{w}_{\alpha}, \nabla \psi\right\rangle_{g} d v_{g} \\
= & \int_{\mathfrak{B}_{2 r_{0}}^{+}\left(x_{\alpha}\right) \backslash \mathfrak{B}_{R \mu_{\alpha}}^{+}\left(x_{\alpha}\right)} \rho^{1-2 \gamma}\left\langle\nabla \hat{w}_{\alpha}, \nabla \psi\right\rangle_{g} d v_{g} \\
& \quad+\int_{\mathfrak{B}_{R \mu_{\alpha}}^{+}\left(x_{\alpha}\right)} \rho^{1-2 \gamma}\left\langle\nabla \hat{w}_{\alpha}, \nabla \psi\right\rangle_{g} d v_{g} \\
= & I_{1}+I_{2} .
\end{aligned}
$$

By Hölder's inequality and the fact that $u \in W^{1,2}\left(\mathbb{R}_{+}^{n+1}, y^{1-2 \gamma}\right)$,

$$
\begin{aligned}
I_{1} \leq & \left(\int_{\mathfrak{B}_{2 r_{0}}^{+}\left(x_{\alpha}\right) \backslash \mathfrak{B}_{R \mu_{\alpha}}^{+}\left(x_{\alpha}\right)} \rho^{1-2 \gamma}\left|\nabla \hat{w}_{\alpha}\right|_{g}^{2} d v_{g}\right)^{\frac{1}{2}} \\
& \times\left(\int_{\mathfrak{B}_{2 r_{0}}^{+}\left(x_{\alpha}\right) \backslash \mathfrak{B}_{R \mu_{\alpha}}^{+}\left(x_{\alpha}\right)} \rho^{1-2 \gamma}|\nabla \psi|_{g}^{2} d v_{g}\right)^{\frac{1}{2}} \\
= & \left(\int_{B_{2 r_{0} \mu_{\alpha}^{-1}}^{+}(0) \backslash B_{R}^{+}(0)} \tilde{\rho}_{\alpha}^{1-2 \gamma}\left|\nabla\left(\tilde{\eta}_{\alpha} u\right)\right|_{\tilde{g}_{\alpha}}^{2} d v_{\tilde{g}_{\alpha}}\right)^{\frac{1}{2}} \\
& \times\left(\int_{\mathfrak{B}_{2 r_{0}}^{+}\left(x_{\alpha}\right) \backslash \mathfrak{B}_{R \mu_{\alpha}}^{+}\left(x_{\alpha}\right)} \rho^{1-2 \gamma}|\nabla \psi|_{g}^{2} d v_{g}\right)^{\frac{1}{2}}=: \beta(R),
\end{aligned}
$$

where

$$
\lim _{R \rightarrow+\infty} \lim _{\alpha \rightarrow+\infty} \sup \beta(R)=0 .
$$

The previous limit is estimated because $u \in W^{1,2}\left(\mathbb{R}_{+}^{n+1}, y^{1-2 \gamma}\right)$, so for any $\alpha, R$,

$$
\left(\int_{B_{2 r_{0} \mu_{\alpha}^{-1}(0) \backslash B_{R}^{+}(0)}^{+}} \tilde{\rho}_{\alpha}^{1-2 \gamma}\left|\nabla\left(\tilde{\eta}_{\alpha} u\right)\right|_{\tilde{g}_{\alpha}}^{2} d v_{\tilde{g}_{\alpha}}\right)^{\frac{1}{2}} \leq C\|u\|_{W^{1,2}\left(\mathbb{R}_{+}^{n+1}, y^{1-2 \gamma}\right)},
$$

and for any $\varepsilon>0$ and any $\alpha$ large, there exists $R_{0}>0$ such that for $R>R_{0}$,

$$
\left(\int_{\mathfrak{B}_{2 r_{0}}^{+}\left(x_{\alpha}\right) \backslash \mathfrak{B}_{R \mu_{\alpha}}^{+}\left(x_{\alpha}\right)} \rho^{1-2 \gamma}|\nabla \psi|_{g}^{2} d v_{g}\right)^{\frac{1}{2}} \leq \varepsilon .
$$


Meanwhile,

$$
\begin{aligned}
I_{2} & \leq\left(\int_{\mathfrak{B}_{R \mu \alpha}^{+}\left(x_{\alpha}\right)} \rho^{1-2 \gamma}\left|\nabla \hat{w}_{\alpha}\right|_{g}^{2} d v_{g}\right)^{\frac{1}{2}}\left(\int_{\mathfrak{B}_{R \mu \alpha}^{+}\left(x_{\alpha}\right)} \rho^{1-2 \gamma}|\nabla \psi|_{g}^{2} d v_{g}\right)^{\frac{1}{2}} \\
& =\left(\int_{B_{R}^{+}(0)} \tilde{\rho}_{\alpha}^{1-2 \gamma}\left|\nabla\left(\tilde{\eta}_{\alpha} u\right)\right|_{\tilde{g}_{\alpha}}^{2} d v_{\tilde{g}_{\alpha}}\right)^{\frac{1}{2}}\left(\int_{\mathfrak{B}_{R \mu_{\alpha}}^{+}\left(x_{\alpha}\right)} \rho^{1-2 \gamma}|\nabla \psi|_{g}^{2} d v_{g}\right)^{\frac{1}{2}}=o(1),
\end{aligned}
$$

uniformly in $R$ as $\alpha \rightarrow+\infty$. To see this, for any $R>0$,

$$
\left(\int_{B_{R}^{+}(0)} \tilde{\rho}_{\alpha}^{1-2 \gamma}\left|\nabla\left(\tilde{\eta}_{\alpha} u\right)\right|_{\tilde{g}_{\alpha}}^{2} d v_{\tilde{g}_{\alpha}}\right)^{\frac{1}{2}} \leq C\|u\|_{W^{1,2}\left(\mathbb{R}_{+}^{n+1}, y^{1-2 \gamma}\right)},
$$

also in Claim 4 we have proved that

$$
\lim _{\alpha \rightarrow+\infty} \mu_{\alpha}=0
$$

and note that $\psi \in W^{1,2}\left(X, \rho^{1-2 \gamma}\right)$. Since $R>0$ is arbitrary, (3-13) implies that

$$
\int_{X} \rho^{1-2 \gamma}\left\langle\nabla \hat{w}_{\alpha}, \nabla \psi\right\rangle_{g} d v_{g}=o(1)
$$

as $\alpha \rightarrow+\infty$.

(ii) For any $\psi \in W^{1,2}\left(X, \rho^{1-2 \gamma}\right)$, the proof of (i) and Propositions 2.4 and 2.6 imply that

$$
d \sigma_{\hat{h}} \rightarrow 0, \quad \text { as } \alpha \rightarrow+\infty .
$$

On the other hand, we have

$$
\begin{aligned}
D I_{g}^{\gamma}\left(\hat{v}_{\alpha}\right) \cdot \psi & =\int_{X} \rho^{1-2 \gamma}\left\langle\nabla \hat{v}_{\alpha}, \nabla \psi\right\rangle_{g} d v_{g}-\int_{M}\left|\hat{v}_{\alpha}\right|^{2^{*}-2} \hat{v}_{\alpha} \psi d \sigma_{\hat{h}} \\
& =D I_{g}^{\gamma}\left(\hat{u}_{\alpha}\right) \cdot \psi-D I_{g}^{\gamma}\left(\hat{w}_{\alpha}\right) \cdot \psi-\int_{M} \Phi_{\alpha} \psi d \sigma_{\hat{h}}
\end{aligned}
$$

where

$$
\Phi_{\alpha}=\left|\hat{u}_{\alpha}-\hat{w}_{\alpha}\right|^{2^{*}-2}\left(\hat{u}_{\alpha}-\hat{w}_{\alpha}\right)+\left|\hat{w}_{\alpha}\right|^{2^{*}-2} \hat{w}_{\alpha}-\left|\hat{u}_{\alpha}\right|^{2^{*}-2} \hat{u}_{\alpha} .
$$

Following the same argument of [Druet et al. 2004, pp. 39-40], we can prove that

$$
\int_{M} \Phi_{\alpha} \psi d \sigma_{\hat{h}} \rightarrow 0, \quad \text { as } \alpha \rightarrow+\infty .
$$

Then, we get that $D I_{g}^{\gamma}\left(\hat{v}_{\alpha}\right) \rightarrow 0$ in $W^{1,2}\left(X, \rho^{1-2 \gamma}\right)^{\prime}$ as $\alpha \rightarrow+\infty$, since $\left\{\hat{u}_{\alpha}\right\}_{\alpha \in \mathbb{N}}$ is a Palais-Smale sequence for $I_{g}^{\gamma}$. 
(iii) Note that $\hat{v}_{\alpha}=\hat{u}_{\alpha}-\hat{w}_{\alpha}$ and $\hat{w}_{\alpha} \equiv 0$ in $X \backslash \mathfrak{B}_{2 r_{0}}^{+}\left(x_{\alpha}\right)$. Given $R>0$, for $\alpha$ large,

$$
\begin{aligned}
& \int_{X} \rho^{1-2 \gamma}\left|\nabla \hat{v}_{\alpha}\right|_{g}^{2} d v_{g} \\
& =\int_{\mathfrak{B}_{2 r_{0}}^{+}\left(x_{\alpha}\right)} \rho^{1-2 \gamma}\left|\nabla \hat{v}_{\alpha}\right|_{g}^{2} d v_{g}+\int_{X \backslash \mathfrak{B}_{2 r_{0}}^{+}\left(x_{\alpha}\right)} \rho^{1-2 \gamma}\left|\nabla \hat{u}_{\alpha}\right|_{g}^{2} d v_{g} \\
& =\int_{\mathfrak{B}_{\mu_{\alpha} R}^{+}\left(x_{\alpha}\right)} \rho^{1-2 \gamma}\left|\nabla \hat{v}_{\alpha}\right|_{g}^{2} d v_{g}+\int_{\mathfrak{B}_{2 r_{0}}^{+}\left(x_{\alpha}\right) \backslash \mathfrak{B}_{\mu_{\alpha} R}^{+}\left(x_{\alpha}\right)} \rho^{1-2 \gamma}\left|\nabla \hat{v}_{\alpha}\right|_{g}^{2} d v_{g} \\
& \quad+\int_{X \backslash \mathfrak{B}_{2 r_{0}}^{+}\left(x_{\alpha}\right)} \rho^{1-2 \gamma}\left|\nabla \hat{u}_{\alpha}\right|_{g}^{2} d v_{g} \\
& =: I_{1}+I_{2}+\int_{X \backslash \mathfrak{B}_{2 r_{0}}^{+}\left(x_{\alpha}\right)} \rho^{1-2 \gamma}\left|\nabla \hat{u}_{\alpha}\right|_{g}^{2} d v_{g} .
\end{aligned}
$$

Since $\tilde{\eta}_{\alpha} \tilde{u}_{\alpha} \rightarrow u$ in $W^{1,2}\left(\mathbb{R}_{+}^{n+1}, y^{1-2 \gamma}\right)$ as $\alpha \rightarrow+\infty$ because of Claim 5,

$$
\begin{aligned}
I_{1} & =\int_{\mathfrak{B}_{\mu_{\alpha}}^{+}\left(x_{\alpha}\right)} \rho^{1-2 \gamma}\left|\nabla\left(\hat{u}_{\alpha}-\hat{w}_{\alpha}\right)\right|_{g}^{2} d v_{g} \\
& =\int_{B_{R}^{+}(0)} \tilde{\rho}_{\alpha}^{1-2 \gamma}\left|\nabla\left(\tilde{u}_{\alpha}-u\right)\right|_{\tilde{g}_{\alpha}}^{2} d v_{\tilde{g}_{\alpha}} \\
& \leq 2 \int_{B_{R}^{+}(0)} y^{1-2 \gamma}\left|\nabla\left(\tilde{u}_{\alpha}-u\right)\right|^{2} d x d y=o(1), \quad \text { as } \alpha \rightarrow+\infty,
\end{aligned}
$$

where we have used that $\tilde{\eta}_{\alpha} \equiv 1$ in $B_{R}^{+}(0)$ for $\alpha$ large.

On the other hand, direct computations give that

$$
\begin{aligned}
\int_{\mathfrak{B}_{2 r_{0}}^{+}\left(x_{\alpha}\right) \backslash \mathfrak{B}_{\mu_{\alpha} R}^{+}\left(x_{\alpha}\right)} \rho^{1-2 \gamma}\left|\nabla \hat{w}_{\alpha}\right|_{g}^{2} d v_{g} & =\int_{B_{2 r_{0} \mu_{\alpha}^{-1}}^{+}(0) \backslash B_{R}^{+}(0)} \tilde{\rho}_{\alpha}^{1-2 \gamma}|\nabla u|_{\tilde{g}_{\alpha}}^{2} d v_{\tilde{g}_{\alpha}} \\
& \leq 2 \int_{B_{2 r_{0} \mu_{\alpha}^{-1}(0) \backslash B_{R}^{+}(0)}^{+}} y^{1-2 \gamma|\nabla u|^{2} d x d y} \\
& =\beta(R),
\end{aligned}
$$

since $u \in W^{1,2}\left(\mathbb{R}_{+}^{n+1}, y^{1-2 \gamma}\right)$ and $\mu_{\alpha} \rightarrow 0$ as $\alpha \rightarrow+\infty$, where $\beta(R)$ is defined as in (3-14). Hence, we get

$$
\begin{aligned}
I_{2} & =\int_{\mathfrak{B}_{2 r_{0}}^{+}\left(x_{\alpha}\right) \backslash \mathfrak{B}_{\mu_{\alpha} R}^{+}\left(x_{\alpha}\right)} \rho^{1-2 \gamma}\left(\left|\nabla \hat{u}_{\alpha}\right|_{g}^{2}+\left|\nabla \hat{w}_{\alpha}\right|_{g}^{2}-2\left\langle\nabla \hat{u}_{\alpha}, \nabla \hat{w}_{\alpha}\right\rangle_{g}\right) d v_{g} \\
& =\int_{\mathfrak{B}_{2 r_{0}}^{+}\left(x_{\alpha}\right) \backslash \mathfrak{B}_{\mu_{\alpha} R}^{+}\left(x_{\alpha}\right)} \rho^{1-2 \gamma}\left|\nabla \hat{u}_{\alpha}\right|_{g}^{2} d v_{g}+\beta(R) .
\end{aligned}
$$


Here we have used Hölder's inequality and the fact that $\left\{\hat{u}_{\alpha}\right\}$ is uniformly bounded in $W^{1,2}\left(X, \rho^{1-2 \gamma}\right)$ to get

$$
\int_{\mathfrak{B}_{2 r_{0}}^{+}\left(x_{\alpha}\right) \backslash \mathfrak{B}_{\mu_{\alpha} R}^{+}\left(x_{\alpha}\right)} \rho^{1-2 \gamma}\left\langle\nabla \hat{u}_{\alpha}, \nabla \hat{w}_{\alpha}\right\rangle_{g} d v_{g}=\beta(R) .
$$

Therefore, noting that $\tilde{u}_{\alpha} \rightarrow u$ in $W^{1,2}\left(\mathbb{R}_{+}^{n+1}, y^{1-2 \gamma}\right)$ as $\alpha \rightarrow+\infty$, by (3-15),

$$
\begin{aligned}
\int_{X} \rho^{1-2 \gamma}\left|\nabla \hat{v}_{\alpha}\right|_{g}^{2} d v_{g} \\
=\int_{X} \rho^{1-2 \gamma}\left|\nabla \hat{u}_{\alpha}\right|_{g}^{2} d v_{g}-\int_{\mathfrak{B}_{\mu_{\alpha}}^{+}\left(x_{\alpha}\right)} \rho^{1-2 \gamma}\left|\nabla \hat{u}_{\alpha}\right|_{g}^{2} d v_{g}+\beta(R)+o(1) \\
=\int_{X} \rho^{1-2 \gamma}\left|\nabla \hat{u}_{\alpha}\right|_{g}^{2} d v_{g}-\int_{B_{R}^{+}(0)} \tilde{\rho}_{\alpha}^{1-2 \gamma}\left|\nabla \tilde{u}_{\alpha}\right|_{\tilde{g}_{\alpha}}^{2} d v_{\tilde{g}_{\alpha}}+\beta(R)+o(1) \\
=\int_{X} \rho^{1-2 \gamma}\left|\nabla \hat{u}_{\alpha}\right|_{g}^{2} d v_{g}-\int_{B_{R}^{+}(0)} y^{1-2 \gamma}|\nabla u|^{2} d x d y+\beta(R)+o(1) \\
=\int_{X} \rho^{1-2 \gamma}\left|\nabla \hat{u}_{\alpha}\right|_{g}^{2} d v_{g}-\int_{\mathbb{R}_{+}^{n+1}} y^{1-2 \gamma}|\nabla u|^{2} d x d y+\beta(R)+o(1) .
\end{aligned}
$$

In a similar way,

$$
\int_{M}\left|\hat{v}_{\alpha}\right|^{2^{*}} d \sigma_{\hat{h}}=\int_{M}\left|\hat{u}_{\alpha}\right|^{2^{*}} d \sigma_{\hat{h}}-\int_{\mathbb{R}^{n}}|u|^{2^{*}} d x+\beta(R)+o(1) .
$$

These imply that

$$
I_{g}^{\gamma}\left(\hat{v}_{\alpha}\right)=I_{g}^{\gamma}\left(\hat{u}_{\alpha}\right)-\widetilde{E}(u)+\beta(R)+o(1) .
$$

Since $R>0$ is arbitrary, we get conclusion (iii).

(iv) It is a direct consequence of (ii) and (iii).

\section{Proof of the main results}

Proof of Theorem 1.3. From Remark 2.10, we have $u_{\alpha} \rightarrow u^{0}$ in $W^{1,2}\left(X, \rho^{1-2 \gamma}\right)$ as $\alpha \rightarrow+\infty$. And $u_{\alpha} \rightarrow u^{0}$ a.e. on $M$ as $\alpha \rightarrow+\infty$. Then, $u^{0} \geq 0$ on $M$ since $u_{\alpha} \geq 0$. Also, $\hat{u}_{\alpha}=u_{\alpha}-u^{0}$ satisfies the Palais-Smale condition and

$$
I_{g}^{\gamma}\left(\hat{u}_{\alpha}\right)=I_{g}^{\gamma, \alpha}\left(u_{\alpha}\right)-I_{g}^{\gamma, \infty}\left(u^{0}\right)+o(1) .
$$

If $\hat{u}_{\alpha} \rightarrow 0$ in $W^{1,2}\left(X, \rho^{1-2 \gamma}\right)$ as $\alpha \rightarrow+\infty$, then the theorem is proved. If $\hat{u}_{\alpha} \rightarrow 0$ but not strongly in $W^{1,2}\left(X, \rho^{1-2 \gamma}\right)$ as $\alpha \rightarrow+\infty$, then, using Lemma 3.1, we can obtain a new Palais-Smale sequence $\left\{\hat{u}_{\alpha}^{1}\right\}_{\alpha \in \mathbb{N}}$ satisfying

$$
I_{g}^{\gamma}\left(\hat{u}_{\alpha}^{1}\right)=I_{g}^{\gamma}\left(\hat{u}_{\alpha}\right)-\widetilde{E}(u)+o(1) .
$$


Now, either $\hat{u}_{\alpha}^{1} \rightarrow 0$ in $W^{1,2}\left(X, \rho^{1-2 \gamma}\right)$ as $\alpha \rightarrow+\infty$, in which case the theorem holds, or $\hat{u}_{\alpha}^{1} \rightarrow 0$ but not strongly in $W^{1,2}\left(X, \rho^{1-2 \gamma}\right)$ as $\alpha \rightarrow+\infty$, in which case we again use Lemma 3.1.

Since $\left\{I_{g}^{\gamma, \alpha}\left(u_{\alpha}\right)\right\}_{\alpha \in \mathbb{N}}$ is uniformly bounded, after a finite number of induction steps, we get the last Palais-Smale sequence (for $m>1$ )

$$
\left\{\hat{u}_{\alpha}^{m}\right\}_{\alpha \in \mathbb{N}} \text { with } I_{g}^{\gamma}\left(\hat{u}_{\alpha}^{m}\right) \rightarrow \beta<\beta_{0}
$$

Then, by Lemma 2.13 , we can get that

$$
\hat{u}_{\alpha}^{m} \rightarrow 0 \quad \text { in } W^{1,2}\left(X, \rho^{2 \gamma-1}\right) \text { as } \alpha \rightarrow+\infty \text {. }
$$

Applying Lemma 3.1 in the process, we can get that $\left\{u^{j}\right\}_{j=1}^{m}$ are solutions to (3-1). We will prove the positivity of $u^{j}, j=1, \ldots, m$, in Lemma 4.2, and the relation (5) of Theorem 1.3 in Lemma 4.1.

For the regularity of $u^{j}$, we can use Lemmas A.1 and A.2.

Lemma 4.1. For any integer $k$ in $[1, m]$, and any integer $l$ in $[0, k-1]$, there exist an integer $s$ and sequences $\left\{y_{\alpha}^{j}\right\}_{\alpha \in \mathbb{N}} \subset M$ and $\left\{\lambda_{\alpha}^{j}>0\right\}_{\alpha \in \mathbb{N}}, j=1, \ldots, s$, such that $d_{h}\left(x_{\alpha}^{k}, y_{\alpha}^{j}\right) / \mu_{\alpha}^{k}$ is bounded, $\lambda_{\alpha}^{j} / \mu_{\alpha}^{k} \rightarrow 0$ as $\alpha \rightarrow+\infty$, and for any $R, R^{\prime}>0$,

$$
\int_{\mathfrak{D}_{R \mu_{\alpha}^{k}}\left(x_{\alpha}^{k}\right) \backslash \bigcup_{j=1}^{s} \mathfrak{D}_{R^{\prime} \lambda_{\alpha}^{j}}\left(y_{\alpha}^{j}\right)}\left|\hat{u}_{\alpha}-\sum_{i=1}^{l} u_{\alpha}^{i}-u_{\alpha}^{k}\right|^{2^{*}} d \sigma_{\hat{h}}=o(1)+\varepsilon\left(R^{\prime}\right),
$$

where

$$
\lim _{R^{\prime} \rightarrow+\infty} \lim _{\alpha \rightarrow+\infty} \sup \varepsilon\left(R^{\prime}\right)=0,
$$

and $\left\{u_{\alpha}^{i}\right\}$ is derived from the rescaling of $u^{i}$ we obtained in the above proof of Theorem 1.3, and $\left\{x_{\alpha}^{i}\right\}$ is the $i$-th likely blow up points sequence.

Proof. We prove this lemma by iteration on $l$. For any integer $k(1 \leq k \leq m)$, if $l=k-1$, then combining the above proof of Theorem 1.3 with Lemma 3.1 and Proposition 2.4,

$$
\int_{\mathfrak{D}_{R \mu_{\alpha}^{k}}\left(x_{\alpha}^{k}\right)}\left|\hat{u}_{\alpha}-\sum_{i=1}^{k-1} u_{\alpha}^{i}-u_{\alpha}^{k}\right|^{2^{*}} d \sigma_{\hat{h}}=o(1),
$$

so (4-1) holds for $s=0$.

Suppose that (4-1) holds for some $l, 1 \leq l \leq k-1$, we need to show that (4-1) holds for $l-1$. 
Case 1: $d \hat{h}\left(x_{\alpha}^{l}, x_{\alpha}^{k}\right) \nrightarrow 0$ as $\alpha \rightarrow+\infty$. Then, for any $\bar{R}>0$, up to a subsequence, $\mathfrak{D}_{\bar{R} \mu_{\alpha}^{l}}\left(x_{\alpha}^{l}\right) \cap \mathfrak{D}_{R \mu_{\alpha}^{k}}\left(x_{\alpha}^{k}\right)=\varnothing$, so we have

$$
\begin{aligned}
\int_{\mathfrak{D}_{R \mu_{\alpha}^{k}}\left(x_{\alpha}^{k}\right) \backslash \cup_{j=1}^{s} \mathfrak{D}_{R^{\prime} \lambda_{\alpha}^{j}}\left(y_{\alpha}^{j}\right)}\left|u_{\alpha}^{l}\right|^{2^{*}} d \sigma_{\hat{h}} & \leq \int_{M \backslash \mathfrak{D}_{\bar{R} \mu_{\alpha}^{l}}\left(x_{\alpha}^{l}\right)}\left|u_{\alpha}^{l}\right|^{2^{*}} d \sigma_{\hat{h}} \\
& \leq C \int_{\mathbb{R}^{n} \backslash D_{\bar{R}(0)}}\left|u^{l}\right|^{2^{*}} d \sigma_{\tilde{h}_{\alpha}} \\
& \leq C \int_{\mathbb{R}^{n} \backslash D_{\bar{R}(0)}}\left|u^{l}\right|^{2^{*}} d x .
\end{aligned}
$$

Since $\bar{R}>0$ is arbitrary and $u^{l} \in L^{2^{*}}\left(\mathbb{R}^{n}\right)$,

$$
\int_{\mathfrak{D}_{R \mu_{\alpha}^{k}}\left(x_{\alpha}^{k}\right) \backslash \bigcup_{j=1}^{s} \mathfrak{D}_{R^{\prime} \lambda_{\alpha}^{j}}\left(y_{\alpha}^{j}\right)}\left|u_{\alpha}^{l}\right|^{2^{*}} d \sigma_{\hat{h}}=o(1), \quad \text { as } \alpha \rightarrow+\infty .
$$

So by the induction hypothesis for $l$ and (4-2), we obtain

$$
\begin{aligned}
\int_{\mathfrak{D}_{R \mu_{\alpha}^{k}}\left(x_{\alpha}^{k}\right) \backslash \bigcup_{j=1}^{s} \mathfrak{D}_{R^{\prime} \lambda_{\alpha}^{j}}\left(y_{\alpha}^{j}\right)}\left|\hat{u}_{\alpha}-\sum_{i=1}^{l-1} u_{\alpha}^{i}-u_{\alpha}^{k}\right|^{2^{*}} d \sigma_{\hat{h}} \\
\leq 2^{2^{*}-1} \int_{\mathfrak{D}_{R \mu_{\alpha}^{k}}\left(x_{\alpha}^{k}\right) \backslash \bigcup_{j=1}^{s} \mathfrak{D}_{R^{\prime} \lambda_{\alpha}^{j}}\left(y_{\alpha}^{j}\right)}\left|\hat{u}_{\alpha}-\sum_{i=1}^{l} u_{\alpha}^{i}-u_{\alpha}^{k}\right|^{2^{*}} d \sigma_{\hat{h}} \\
\quad+2^{2^{*}-1} \int_{\mathfrak{D}_{R \mu_{\alpha}^{k}}\left(x_{\alpha}^{k}\right) \backslash \bigcup_{j=1}^{s} \mathfrak{D}_{R^{\prime} \lambda_{\alpha}^{j}}\left(y_{\alpha}^{j}\right)}\left|u_{\alpha}^{l}\right|^{2^{*}} d \sigma_{\hat{h}} \\
=o(1)+\varepsilon\left(R^{\prime}\right) .
\end{aligned}
$$

Thus we have proven that (4-1) holds for $l-1$.

Case 2: $d \hat{h}\left(x_{\alpha}^{l}, x_{\alpha}^{k}\right) \rightarrow 0$ as $\alpha \rightarrow+\infty$. Let $r_{0}$ be sufficiently small such that for any $P \in M, x, y \in \mathbb{R}^{n}$, and $|x|,|y| \leq r_{0}$,

$$
\frac{1}{2}|x-y| \leq d \hat{h}\left(\varphi_{P}(x), \varphi_{P}(y)\right) \leq 2|x-y| .
$$

Let $\tilde{x}_{\alpha}^{l}=\left(\mu_{\alpha}^{k}\right)^{-1} \varphi_{x_{\alpha}^{k}}^{-1}\left(x_{\alpha}^{l}\right)$ and $\tilde{y}_{\alpha}^{j}=\left(\mu_{\alpha}^{k}\right)^{-1} \varphi_{x_{\alpha}^{k}}^{-1}\left(y_{\alpha}^{j}\right)$. Then,

$$
\left\{\begin{array}{l}
D_{\frac{R}{2} \mu_{\alpha}^{l} / \mu_{\alpha}^{k}}\left(\tilde{x}_{\alpha}^{l}\right) \subset\left(\mu_{\alpha}^{k}\right)^{-1} \varphi_{x_{\alpha}^{k}}^{-1}\left(\mathfrak{D}_{R \mu_{\alpha}^{l}}\left(x_{\alpha}^{l}\right)\right) \subset D_{2 R \mu_{\alpha}^{l} / \mu_{\alpha}^{k}}\left(\tilde{x}_{\alpha}^{l}\right), \\
D_{\frac{R}{2} \lambda_{\alpha}^{j} / \mu_{\alpha}^{k}}\left(\tilde{y}_{\alpha}^{j}\right) \subset\left(\mu_{\alpha}^{k}\right)^{-1} \varphi_{x_{\alpha}^{k}}^{-1}\left(\mathfrak{D}_{R \lambda_{\alpha}^{j}}\left(y_{\alpha}^{j}\right)\right) \subset D_{2 R \lambda_{\alpha}^{j} / \mu_{\alpha}^{k}}\left(\tilde{y}_{\alpha}^{j}\right) .
\end{array}\right.
$$

Given $\tilde{R}>0$, from Lemma 3.1, Proposition 2.4, and the proof of Theorem 1.3,

$$
\int_{\mathfrak{D}_{\tilde{R} \mu_{\alpha}^{l}}\left(x_{\alpha}^{l}\right)}\left|\hat{u}_{\alpha}-\sum_{i=1}^{l} u_{\alpha}^{i}\right|^{2^{*}} d \sigma_{\hat{h}}=o(1) .
$$


By the assumption for $1 \leq l \leq k-1$, i.e.,

$$
\int_{\mathfrak{D}_{R \mu_{\alpha}^{k}}\left(x_{\alpha}^{k}\right) \backslash \cup_{j=1}^{s} \mathfrak{D}_{R^{\prime} \lambda_{\alpha}^{j}}\left(y_{\alpha}^{j}\right)}\left|\hat{u}_{\alpha}-\sum_{i=1}^{l} u_{\alpha}^{i}-u_{\alpha}^{k}\right|^{2^{*}} d \sigma_{\hat{h}}=o(1)+\varepsilon\left(R^{\prime}\right),
$$

combined with (4-4),

$$
\int_{\left[\mathfrak{D}_{R \mu_{\alpha}^{k}}\left(x_{\alpha}^{k}\right) \backslash \bigcup_{j=1}^{s} \mathfrak{D}_{R^{\prime} \lambda_{\alpha}^{j}}\left(y_{\alpha}^{j}\right)\right] \cap \mathfrak{D}_{\tilde{R} \mu_{\alpha}^{l}}\left(x_{\alpha}^{l}\right)}\left|u_{\alpha}^{k}\right|^{2^{*}} d \sigma_{\hat{h}}=o(1)+\varepsilon\left(R^{\prime}\right),
$$

so using (4-3) we arrive at

$$
\int_{\left[D_{R}(0) \backslash \bigcup_{j=1}^{s} D_{2 R^{\prime} \lambda_{\alpha}^{j} / \mu_{\alpha}^{k}}\left(\tilde{y}_{\alpha}^{j}\right)\right] \cap D_{1 / 2 \tilde{R} \mu_{\alpha}^{l} / \mu_{\alpha}^{k}}\left(\tilde{x}_{\alpha}^{l}\right)}\left|u^{k}\right|^{2^{*}} d \sigma_{\tilde{h}_{\alpha}}=o(1)+\varepsilon\left(R^{\prime}\right) .
$$

Next, we consider two scenarios: first, assume that $d \hat{h}\left(x_{\alpha}^{l}, x_{\alpha}^{k}\right) / \mu_{\alpha}^{k} \rightarrow+\infty$ as $\alpha \rightarrow+\infty$. We claim that $d_{\hat{h}}\left(x_{\alpha}^{l}, x_{\alpha}^{k}\right) / \mu_{\alpha}^{l} \rightarrow+\infty$ as $\alpha \rightarrow+\infty$. If not, then (4-5) with $\tilde{R}$ large enough yields that $\mu_{\alpha}^{l} / \mu_{\alpha}^{k} \rightarrow 0$ as $\alpha \rightarrow+\infty$. Moreover,

$$
\frac{d \hat{h}\left(x_{\alpha}^{l}, x_{\alpha}^{k}\right)}{\mu_{\alpha}^{l}}=\frac{d \hat{h}\left(x_{\alpha}^{l}, x_{\alpha}^{k}\right)}{\mu_{\alpha}^{k}} \frac{\mu_{\alpha}^{k}}{\mu_{\alpha}^{l}},
$$

so we can choose $\tilde{R}>0$ such that $\mathfrak{D}_{\tilde{R} \mu_{\alpha}^{k}}\left(x_{\alpha}^{k}\right) \cap \mathfrak{D}_{\tilde{R} \mu_{\alpha}^{l}}\left(x_{\alpha}^{l}\right)=\varnothing$, which reduces to the previous Case 1; as a consequence, (4-1) holds for $l-1$.

Second, if $d \hat{h}\left(x_{\alpha}^{l}, x_{\alpha}^{k}\right) / \mu_{\alpha}^{k} \nrightarrow+\infty$ as $\alpha \rightarrow+\infty$, then, up to a subsequence, $d \hat{h}\left(x_{\alpha}^{l}, x_{\alpha}^{k}\right) / \mu_{\alpha}^{k}$ converges. So, (4-5) implies that $\mu_{\alpha}^{l} / \mu_{\alpha}^{k} \rightarrow+\infty$. Set $y_{\alpha}^{s+1}=x_{\alpha}^{l}$ and $\lambda_{\alpha}^{s+1}=\mu_{\alpha}^{l}$. Then,

$$
\int_{\mathfrak{D}_{R \mu_{\alpha}^{k}}\left(x_{\alpha}^{k}\right) \backslash \cup_{j=1}^{s+1} \mathfrak{D}_{R^{\prime} \lambda_{\alpha}^{j}}\left(y_{\alpha}^{j}\right)}\left|\hat{u}_{\alpha}-\sum_{i=1}^{l} u_{\alpha}^{i}-u_{\alpha}^{k}\right|^{2^{*}} d \sigma_{\hat{h}}=o(1)+\varepsilon\left(R^{\prime}\right)
$$

and

$$
\begin{aligned}
\int_{\mathfrak{D}_{R \mu_{\alpha}^{k}}\left(x_{\alpha}^{k}\right) \backslash \bigcup_{j=1}^{s+1} \mathfrak{D}_{R^{\prime} \lambda_{\alpha}^{j}}\left(y_{\alpha}^{j}\right)}\left|u_{\alpha}^{l}\right|^{2^{*}} d \sigma_{\hat{h}} & \leq \int_{M \backslash \mathfrak{D}_{R^{\prime} \mu_{\alpha}^{l}}^{l}\left(x_{\alpha}^{l}\right)}\left|u_{\alpha}^{l}\right|^{2^{*}} d \sigma_{\hat{h}} \\
& \leq C \int_{\mathbb{R}^{n} \backslash D_{R^{\prime}}(0)}\left|u^{l}\right|^{2^{*}} d x \leq \varepsilon\left(R^{\prime}\right),
\end{aligned}
$$

which yield that

$$
\int_{\mathfrak{D}_{R \mu_{\alpha}^{k}}\left(x_{\alpha}^{k}\right) \backslash \cup_{j=1}^{s+1} \mathfrak{D}_{R^{\prime} \lambda_{\alpha}^{j}}\left(y_{\alpha}^{j}\right)}\left|\hat{u}_{\alpha}-\sum_{i=1}^{l-1} u_{\alpha}^{i}-u_{\alpha}^{k}\right|^{2^{*}} d \sigma_{\hat{h}}=o(1)+\varepsilon\left(R^{\prime}\right) .
$$

In particular, (4-1) holds for $l-1$, as desired. The iteration process is thus completed. 
Moreover, we have also shown that for any $i \neq j$

$$
\frac{\mu_{\alpha}^{i}}{\mu_{\alpha}^{j}}+\frac{\mu_{\alpha}^{j}}{\mu_{\alpha}^{i}}+\frac{d \hat{h}\left(x_{\alpha}^{i}, x_{\alpha}^{j}\right)^{2}}{\mu_{\alpha}^{i} \mu_{\alpha}^{j}} \rightarrow+\infty \quad \text { as } \alpha \rightarrow+\infty ;
$$

compare [Almaraz 2014; Druet et al. 2004; Struwe 1984]. Note that this convergence contains two kinds of bubbles: one case is that $\mu_{\alpha}^{i}=O\left(\mu_{\alpha}^{j}\right)$ when $\alpha \rightarrow+\infty$; then the two blow up points are far away from each other. The other case is that $\mu_{\alpha}^{i}=o\left(\mu_{\alpha}^{j}\right)$ or $\mu_{\alpha}^{j}=o\left(\mu_{\alpha}^{i}\right)$ when $\alpha \rightarrow+\infty$; then the distance of the two blow up point cannot be determined. Also we get that $\lambda_{\alpha}^{j} / \mu_{\alpha}^{k} \rightarrow 0$ as $\alpha \rightarrow+\infty$.

Lemma 4.2. The $u^{i}$ (for $\left.i=0,1, \ldots, m\right)$ that we get in the Theorem 1.3 are all nonnegative. In particular, for $i \geq 1, u^{i}$ is of the form $U_{a_{i}}^{\lambda_{i}}$ for some $\lambda_{i}>0$ and $a_{i} \in \mathbb{R}^{n}$, where $U_{a_{i}}^{\lambda_{i}}$ is as in (1-13).

Proof. First of all, note that $u^{0} \geq 0$ in $\bar{X}$ by Proposition 2.11. So, we just need to prove the positivity of $u^{i}$ for $i \geq 1$. For any $k \in[1, m]$, taking $l=0$ in Lemma 4.1,

$$
\int_{\mathfrak{D}_{R \mu_{\alpha}^{k}}\left(x_{\alpha}^{k}\right) \backslash \bigcup_{j=1}^{s} \mathfrak{D}_{R^{\prime} \lambda_{\alpha}^{j}}\left(y_{\alpha}^{j}\right)}\left|\hat{u}_{\alpha}-U_{\alpha}^{k}\right|^{2^{*}} d \sigma_{\hat{h}}=o(1)+\varepsilon\left(R^{\prime}\right)
$$

where

$$
U_{\alpha}^{k}(x)=\left(\mu_{\alpha}^{k}\right)^{-\frac{n-2 \gamma}{2}} u^{k}\left(\left(\mu_{\alpha}^{k}\right)^{-1} \varphi_{x_{\alpha}^{k}}^{-1}(x)\right) \quad \text { for } x \in \mathfrak{D}_{R \mu_{\alpha}^{k}}\left(x_{\alpha}^{k}\right)
$$

is called a bubble. Since $u_{\alpha}=\hat{u}_{\alpha}+u^{0}$, for $x \in D_{r_{0} / \mu_{\alpha}^{k}}(0) \subset \mathbb{R}^{n}$, where $r_{0}$ is the same as the one mentioned in Theorem 1.3,

$$
u_{\alpha}^{k}(x)=\tilde{u}_{\alpha}^{k}(x)+\tilde{u}_{\alpha}^{0, k}(x),
$$

where

$$
\begin{gathered}
u_{\alpha}^{k}(x)=\left(\mu_{\alpha}^{k}\right)^{\frac{n-2 \gamma}{2}} u_{\alpha}\left(\varphi_{x_{\alpha}^{k}}\left(\mu_{\alpha}^{k} x\right)\right), \\
\tilde{u}_{\alpha}^{k}(x)=\left(\mu_{\alpha}^{k}\right)^{\frac{n-2 \gamma}{2}} \hat{u}_{\alpha}\left(\varphi_{x_{\alpha}^{k}}\left(\mu_{\alpha}^{k} x\right)\right), \\
\tilde{u}_{\alpha}^{0, k}(x)=\left(\mu_{\alpha}^{k}\right)^{\frac{n-2 \gamma}{2}} u^{0}\left(\varphi_{x_{\alpha}^{k}}\left(\mu_{\alpha}^{k} x\right)\right) .
\end{gathered}
$$

Then, (4-6) implies that

$$
\int_{D_{R}(0) \backslash \bigcup_{j=1}^{s} D_{2 R^{\prime} \lambda_{\alpha}^{j} / \mu_{\alpha}^{k}}\left(\tilde{y}_{\alpha}^{j}\right)}\left|\tilde{u}_{\alpha}^{k}-u^{k}\right|^{2^{*}} d x=o(1)+\varepsilon\left(R^{\prime}\right),
$$

where $\tilde{y}_{\alpha}^{j}=\left(\mu_{\alpha}^{k}\right)^{-1} \varphi_{x_{\alpha}^{k}}^{-1}\left(y_{\alpha}^{j}\right)$. Since $\left\{d \hat{h}\left(x_{\alpha}^{k}, y_{\alpha}^{j}\right) / \mu_{\alpha}^{k}\right\}_{\alpha \in \mathbb{N}}$ is uniformly bounded by Lemma 4.1, $\left\{\tilde{y}_{\alpha}^{j}\right\}_{\alpha \in \mathbb{N}}$ is bounded and there exists a subsequence, also denoted by $\left\{\tilde{y}_{\alpha}^{j}\right\}$, such that $\tilde{y}_{\alpha}^{j} \rightarrow \tilde{y}^{j}$ as $\alpha \rightarrow+\infty$ for $j=1, \ldots, s$. Combining (4-7) with $\lambda_{\alpha}^{j} / \mu_{\alpha}^{k} \rightarrow 0$ as $\alpha \rightarrow+\infty$, we get

$$
\tilde{u}_{\alpha}^{k} \rightarrow u^{k} \quad \text { in } L_{\mathrm{loc}}^{2^{*}}\left(D_{R}(0) \backslash Y\right),
$$


as $\alpha \rightarrow+\infty$ for $Y=\left\{\tilde{y}^{j}\right\}_{j=1}^{s}$, so

$$
\tilde{u}_{\alpha}^{k} \rightarrow u^{k} \quad \text { a.e. in } \mathbb{R}^{n},
$$

since $R>0$ is arbitrary.

Also note that

$$
\int_{\mathfrak{D}_{R \mu_{\alpha}^{k}}\left(x_{\alpha}^{k}\right)}\left|u^{0}\right|^{2^{*}} d \sigma_{\hat{h}}=\int_{D_{R}(0)}\left|\tilde{u}_{\alpha}^{0, k}\right|^{2^{*}} d \sigma_{\tilde{h}_{\alpha}^{k}},
$$

where $\tilde{h}_{\alpha}^{k}(x)=\left(\varphi_{x_{\alpha}^{k}}^{*} \hat{h}\right)\left(\mu_{\alpha}^{k} x\right)$. Then, $\mu_{\alpha}^{k} \rightarrow 0$ as $\alpha \rightarrow+\infty$ and $u^{0} \in L^{2^{*}}(M, \hat{h})$ yield that

$$
\tilde{u}_{\alpha}^{0, k} \rightarrow 0, \quad \text { in } L^{2^{*}}\left(D_{R}(0),|d x|^{2}\right)
$$

as $\alpha \rightarrow+\infty$, so

$$
\tilde{u}_{\alpha}^{0, k} \rightarrow 0 \quad \text { a.e. in } \mathbb{R}^{n}
$$

since $R>0$ is arbitrary.

In particular, we have shown that $u_{\alpha}^{k} \rightarrow u^{k}$ almost everywhere on $\mathbb{R}^{n}$ as $\alpha \rightarrow+\infty$. Note that $u_{\alpha}$ is nonnegative by definition, so $u_{\alpha}^{k} \geq 0$ on $\mathbb{R}^{n}$. We conclude that $u^{k} \geq 0$ on $\mathbb{R}^{n}$. Then by the maximum principle, it follows that $u^{k} \geq 0$ in $\mathbb{R}_{+}^{n+1}$. Due to the previous arguments, $u^{k}$ is of the form $U_{a_{k}}^{\lambda_{k}}$ for some $\lambda_{k}>0$ and $a_{k} \in \mathbb{R}^{n}$, where $U_{a_{k}}^{\lambda_{k}}$ is as in (1-13).

\section{Appendix}

We will prove the $\mathscr{C}^{\infty}$ estimates from the $L^{\infty}$ estimates by the Harnack inequality. The two important lemmas are given here.

Lemma A.1 [González and Qing 2013]. Let $R>0$ and $u$ be a weak solution of

$$
\begin{cases}-\operatorname{div}\left(y^{1-2 \gamma} \nabla u\right)=0 & \text { in } B_{2 R}^{+}(0), \\ -\lim _{y \rightarrow 0} y^{1-2 \gamma} \partial_{y} u=f(x) u+g(x)|u|^{2^{*}-2} u & \text { on } D_{2 R}(0) .\end{cases}
$$

Here, $f$ and $g$ are smooth functions on $D_{2 R}(0)$. Assume that

$$
\lambda=\int_{D_{2 R}(0)}|u|^{2^{*}} d x<\infty .
$$

Then, for any $p>1$, there exists a constant $C_{p}=C(p, \lambda)$ such that

$$
\sup _{B_{R}^{+}(0)}|u|+\sup _{D_{R}(0)}|u| \leq C_{p}\left(R^{-\frac{n+2-2 \gamma}{p}}\|u\|_{L^{p}\left(B_{2 R}^{+}(0)\right)}+R^{-\frac{n}{p}}\|u\|_{L^{p}\left(D_{2 R}(0)\right)}\right) .
$$


Lemma A.2 [Jin et al. 2014]. Let $a(x), b(x) \in \mathscr{C}^{\alpha}\left(D_{2}(0)\right)$ for some $0<\alpha \notin \mathbb{N}$ and let $u \in W^{1,2}\left(B_{2}^{+}(0), y^{1-2 \gamma}\right)$ be a weak solution of

$$
\begin{cases}-\operatorname{div}\left(y^{1-2 \gamma} \nabla u\right)=0 & \text { in } B_{2}^{+}(0), \\ -\lim _{y \rightarrow 0} y^{1-2 \gamma} \partial_{y} u=a(x) u+b(x) & \text { on } D_{2}(0) .\end{cases}
$$

If $2 \gamma+\alpha \notin \mathbb{N}$, then $u(\cdot, 0)$ is of $\mathfrak{C}^{2 \gamma+\alpha}\left(D_{1}(0)\right)$, and

$$
\|u(\cdot, 0)\|_{C^{2 \gamma+\alpha}\left(D_{1}(0)\right)} \leq C\left(\|u\|_{L^{\infty}\left(B_{2}^{+}(0)\right)}+\|b\|_{C^{\alpha}\left(D_{2}(0)\right)}\right)
$$

where $C>0$ depends only on $n, \gamma, \alpha$, and $\|a\|_{\mathscr{C}^{\alpha}\left(D_{2}(0)\right)}$.

\section{Acknowledgments}

The authors thank Professor Jie Qing for useful discussions and comments on this work.

\section{References}

[Almaraz 2014] S. Almaraz, "The asymptotic behavior of Palais-Smale sequences on manifolds with boundary”, Pacific J. Math. 269:1 (2014), 1-17. MR 3233907 Zbl 1307.35116

[Caffarelli and Silvestre 2007] L. Caffarelli and L. Silvestre, "An extension problem related to the fractional Laplacian”, Comm. Partial Differential Equations 32 (2007), 1245-1260. MR 2009k:35096 Zbl 1143.26002

[Case 2015] J. S. Case, "Some energy inequalities involving fractional GJMS operators", preprint, 2015. arXiv 1509.08347

[Case and Chang 2015] J. S. Case and S.-Y. A. Chang, "On fractional GJMS operators", Comm. Pure Appl. Math. (online publication February 2015).

[Chang and González 2011] S.-Y. A. Chang and M. d. M. González, "Fractional Laplacian in conformal geometry", Adv. Math. 226:2 (2011), 1410-1432. MR 2012k:58057 Zbl 1214.26005

[Di Nezza et al. 2012] E. Di Nezza, G. Palatucci, and E. Valdinoci, "Hitchhiker's guide to the fractional Sobolev spaces", Bull. Sci. Math. 136:5 (2012), 521-573. MR 2944369 Zbl 1252.46023

[Druet et al. 2004] O. Druet, E. Hebey, and F. Robert, Blow-up theory for elliptic PDEs in Riemannian geometry, Mathematical Notes 45, Princeton University Press, 2004. MR 2005g:53058 Zbl 1059.58017

[González 2015] M. d. M. González, "Further results on the fractional Yamabe problem: the umbilic case", preprint, 2015. arXiv 1503.02862v1

[González and Qing 2013] M. d. M. González and J. Qing, "Fractional conformal Laplacians and fractional Yamabe problems", Anal. PDE 6:7 (2013), 1535-1576. MR 3148060 Zbl 1287.35039

[Graham and Zworski 2003] C. R. Graham and M. Zworski, "Scattering matrix in conformal geometry”, Invent. Math. 152:1 (2003), 89-118. MR 2004c:58064 Zbl 1030.58022

[Graham et al. 1992] C. R. Graham, R. Jenne, L. J. Mason, and G. A. J. Sparling, "Conformally invariant powers of the Laplacian, I: Existence", J. London Math. Soc. (2) 46:3 (1992), 557-565. MR 94c:58226 Zbl 0726.53010

[Jin and Xiong 2013] T. Jin and J. Xiong, "Sharp constants in weighted trace inequalities on Riemannian manifolds", Calc. Var. Partial Differential Equations 48:3-4 (2013), 555-585. MR 3116023 Zbl 1288.46026 
[Jin et al. 2014] T. Jin, Y. Li, and J. Xiong, "On a fractional Nirenberg problem, I: Blow up analysis and compactness of solutions", J. Eur. Math. Soc. (JEMS) 16:6 (2014), 1111-1171. MR 3226738 Zbl 1300.53041

[Kufner 1985] A. Kufner, Weighted Sobolev spaces, Wiley, New York, 1985. MR 86m:46033 Zbl 0567.46009

[Palatucci and Pisante 2014] G. Palatucci and A. Pisante, "Improved Sobolev embeddings, profile decomposition, and concentration-compactness for fractional Sobolev spaces", Calc. Var. Partial Differential Equations 50:3-4 (2014), 799-829. MR 3216834 Zbl 1296.35064

[Palatucci and Pisante 2015] G. Palatucci and A. Pisante, "A global compactness type result for PalaisSmale sequences in fractional Sobolev spaces", Nonlinear Anal. 117 (2015), 1-7. MR 3316602 Zbl 1312.35092

[Paneitz 2008] S. M. Paneitz, "A quartic conformally covariant differential operator for arbitrary pseudo-Riemannian manifolds (summary)", SIGMA Symmetry Integrability Geom. Methods Appl. 4 (2008), Paper 036, 3. MR 2009b:58080 Zbl 1145.53053

[Struwe 1984] M. Struwe, "A global compactness result for elliptic boundary value problems involving limiting nonlinearities”, Math. Z. 187:4 (1984), 511-517. MR 86k:35046 Zbl 0535.35025

[Yan et al. 2015] S. Yan, J. Yang, and X. Yu, "Equations involving fractional Laplacian operator: compactness and application", J. Funct. Anal. 269:1 (2015), 47-79. MR 3345604 Zbl 1317.35287

Received October 20, 2014.

YI FANG

SCHOOL OF Mathematical SCIENCES

UNIVERSITY OF SCIENCE AND TECHNOLOGY OF CHINA

GUANLI-KEYAN BUILDING

96 JINZHAI ROAD, HEFEI

230026 ANHUI

CHINA

fangyi1915@gmail.com

MARÍA DEL MAR GONZÁLEZ

ETSEIB - DEPARTAMENT DE MATEMATICA APLICADA I

Universitat Politecnica de Catalunya

AVENIDA DIAGONAL, 647

08028 BARCELONA

SPAIN

mar.gonzalez@upc.edu 


\title{
PACIFIC JOURNAL OF MATHEMATICS
}

\author{
msp.org/pjm
}

Founded in 1951 by E. F. Beckenbach (1906-1982) and F. Wolf (1904-1989)

\section{EDITORS}

Don Blasius (Managing Editor)

Department of Mathematics

University of California

Los Angeles, CA 90095-1555

blasius@math.ucla.edu

\author{
Paul Balmer \\ Department of Mathematics \\ University of California \\ Los Angeles, CA 90095-1555 \\ balmer@math.ucla.edu \\ Robert Finn \\ Department of Mathematics \\ Stanford University \\ Stanford, CA 94305-2125 \\ finn@math.stanford.edu \\ Sorin Popa \\ Department of Mathematics \\ University of California \\ Los Angeles, CA 90095-1555 \\ popa@math.ucla.edu
}

\author{
Vyjayanthi Chari \\ Department of Mathematics \\ University of California \\ Riverside, CA 92521-0135 \\ chari@math.ucr.edu \\ Kefeng Liu \\ Department of Mathematics \\ University of California \\ Los Angeles, CA 90095-1555 \\ liu@math.ucla.edu \\ Jie Qing \\ Department of Mathematics \\ University of California \\ Santa Cruz, CA 95064 \\ qing@ cats.ucsc.edu
}

\section{PRODUCTION}

Silvio Levy, Scientific Editor, production@msp.org

\section{SUPPORTING INSTITUTIONS}

ACADEMIA SINICA, TAIPEI

CALIFORNIA INST. OF TECHNOLOGY

INST. DE MATEMÁTICA PURA E APLICADA

KEIO UNIVERSITY

MATH. SCIENCES RESEARCH INSTITUTE

NEW MEXICO STATE UNIV.

OREGON STATE UNIV.

\author{
STANFORD UNIVERSITY \\ UNIV. OF BRITISH COLUMBIA \\ UNIV. OF CALIFORNIA, BERKELEY \\ UNIV. OF CALIFORNIA, DAVIS \\ UNIV. OF CALIFORNIA, LOS ANGELES \\ UNIV. OF CALIFORNIA, RIVERSIDE \\ UNIV. OF CALIFORNIA, SAN DIEGO \\ UNIV. OF CALIF., SANTA BARBARA
}

\author{
Daryl Cooper \\ Department of Mathematics \\ University of California \\ Santa Barbara, CA 93106-3080 \\ cooper@math.ucsb.edu \\ Jiang-Hua Lu \\ Department of Mathematics \\ The University of Hong Kong \\ Pokfulam Rd., Hong Kong \\ jhlu@maths.hku.hk \\ Paul Yang \\ Department of Mathematics \\ Princeton University \\ Princeton NJ 08544-1000 \\ yang@math.princeton.edu
}

These supporting institutions contribute to the cost of publication of this Journal, but they are not owners or publishers and have no responsibility for its contents or policies.

See inside back cover or msp.org/pjm for submission instructions.

The subscription price for 2015 is US \$420/year for the electronic version, and \$570/year for print and electronic.

Subscriptions, requests for back issues and changes of subscribers address should be sent to Pacific Journal of Mathematics, P.O. Box 4163, Berkeley, CA 94704-0163, U.S.A. The Pacific Journal of Mathematics is indexed by Mathematical Reviews, Zentralblatt MATH, PASCAL CNRS Index, Referativnyi Zhurnal, Current Mathematical Publications and Web of Knowledge (Science Citation Index).

The Pacific Journal of Mathematics (ISSN 0030-8730) at the University of California, c/o Department of Mathematics, 798 Evans Hall \#3840, Berkeley, CA 94720-3840, is published twelve times a year. Periodical rate postage paid at Berkeley, CA 94704, and additional mailing offices. POSTMASTER: send address changes to Pacific Journal of Mathematics, P.O. Box 4163, Berkeley, CA 94704-0163.

PJM peer review and production are managed by EditFLOW ${ }^{\circledR}$ from Mathematical Sciences Publishers.

\section{PUBLISHED BY}

\section{mathematical sciences publishers \\ nonprofit scientific publishing}

http://msp.org/

(C) 2015 Mathematical Sciences Publishers 


\section{PACIFIC JOURNAL OF MATHEMATICS}

Volume $278 \quad$ No. $2 \quad$ December 2015

Differential Harnack and logarithmic Sobolev inequalities along

Ricci-harmonic map flow

ABIMBOLA ABOLARINWA

On $J$-holomorphic curves in almost complex manifolds with

291

asymptotically cylindrical ends

ERKAO BAO

Integration of coupling Dirac structures

OLIVIER BRAHIC and RUI LOJA FERNANDES

Asymptotic behavior of Palais-Smale sequences associated with fractional Yamabe-type equations

Yi FANG and MARÍA DEL MAR GONZÁLEZ

$K$-theory and homotopies of 2-cocycles on higher-rank graphs

ELIZABETH GILLASPY

Fusion products and toroidal algebras

DENIZ KUS and PETER LiTTELMANN

Differential Harnack estimates for positive solutions to heat equation under Finsler-Ricci flow

\section{SAJJAD LAKZIAN}

On the one-endedness of graphs of groups

NichOLAS TOUIKAN

On the structure of vertex cuts separating the ends of a graph

GARETH R. WILKES 